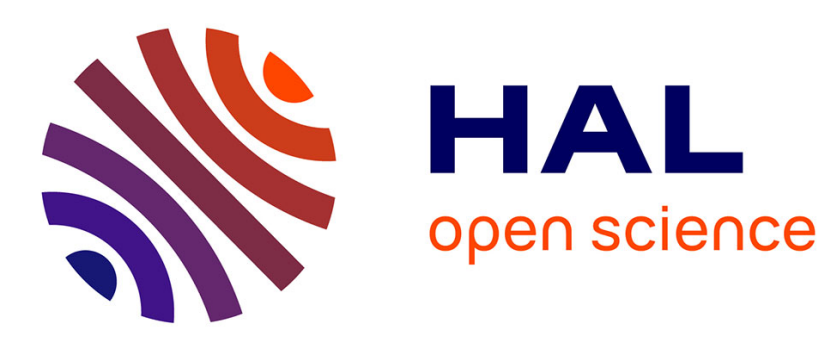

\title{
On the control of finite-dimensional mechanical systems with unilateral constants
}

\author{
Bernard Brogliato, Silviu-Iulian Niculescu, Pascal Orhant
}

\section{To cite this version:}

Bernard Brogliato, Silviu-Iulian Niculescu, Pascal Orhant. On the control of finite-dimensional mechanical systems with unilateral constants. IEEE Transactions on Automatic Control, 1997, 42 (2), pp.200-215. 10.1109/9.554400 . hal-02301675

\section{HAL Id: hal-02301675 \\ https://hal.science/hal-02301675}

Submitted on 6 Nov 2019

HAL is a multi-disciplinary open access archive for the deposit and dissemination of scientific research documents, whether they are published or not. The documents may come from teaching and research institutions in France or abroad, or from public or private research centers.
L'archive ouverte pluridisciplinaire $\mathbf{H A L}$, est destinée au dépôt et à la diffusion de documents scientifiques de niveau recherche, publiés ou non, émanant des établissements d'enseignement et de recherche français ou étrangers, des laboratoires publics ou privés. 


\title{
On the Control of Finite-Dimensional Mechanical Systems with Unilateral Constraints
}

\author{
Bernard Brogliato, Silviu-Iulian Niculescu, and Pascal Orhant
}

\begin{abstract}
This paper focuses on the problem of the control of a class of mechanical systems with a finite number of degrees-offreedom, subject to unilateral constraints on the position. Roughly speaking, those systems are described by a set of ordinary differential equations that represent smooth dynamics, together with an algebraic inequality condition $F(q) \geq 0$ (where $q$ is the vector of generalized coordinates) and an impact rule relating the interaction impulse and the velocity. Nonsmooth dynamics is at the core of the study of such systems. This implies one can suitably define solutions and stability concepts that fit with the considered model. Then, we discuss the closed-loop control problem, and we analyze various switching control strategies.
\end{abstract}

Index Terms - Closed-loop stability, mechanical systems, switching control, unilateral constraints.

\section{INTRODUCTION}

$\mathbf{T}$ THE LAST fifteen years have witnessed a considerable interest in the problem of control of mechanical systems. Both motion control and hybrid force/position control cases have received much attention. In the first case, the system is assumed to evolve in a space free of obstacles and is described by a set of ordinary differential equations (ODE). Feedback linearization as well as more specific controllers (adaptive, robust control) have been proposed [29], [30]. In the second case, the system is assumed to evolve on a constraint submanifold of the form $F(q)=0$. Interaction forces between the manipulator's tip and the obstacle have to be taken into account in the analysis. Solutions based on a decoupling between free tangential motion along the surface $F(q)=0$ and the constraint normal direction have been proposed [5], [7]. It is worth noting that in this latter case, the constraint is assumed to be verified for all times without any consideration for the possible transition between configurations $q$ such that $F(q)>0$ and such that $F(q)=$ 0 . This has considerable consequences, since the system's trajectories can still be considered as smooth time functions. Hence all results available on existence, uniqueness, and stability can be applied directly to such systems.

In parallel with studies devoted to systems of rigid bodies with unilateral constraints and linear complementarity prob-

B. Brogliato and P. Orhant are with the Laboratoire d'Automatique de Grenoble (UMR 5528 CNRS-INPG), ENSIEG, BP 46, 38402 St.-Martind'Hères, France. They are also with the GDR CNRS "Automatique."

S.-I. Niculescu is with the Laboratoire d'Automatique de Grenoble (UMR 5528 CNRS-INPG), ENSIEG, BP 46, 38402 St.-Martin-d'Hères, France. He is also with the GDR CNRS "Automatique," on leave from the Department of Automatic Control, University "Politehnica" Bucharest, Romania. lems [60], [61], there has recently been a growing interest in the modeling of collisions in kinematic chains [46]-[49] and the control of complete robotic tasks, i.e., tasks involving freemotion as well as constrained motion phases like deburring and grinding operations. Although this is not too common yet, it is quite possible that manipulators may carry out such tasks as driving nails, compacting powders, breaking objects ..., in which case they would be intended to be impacters [8] (it is also worth noting that all mechanisms with clearances involve impact dynamics). Many experimental works have been devoted to studying the transition phase control, which occurs when the robot's tip strikes the environment's surface; see, for instance, [19]-[23], [42], [44], [55], [56], and [59]. In particular, the results in [44] (flexible environment) and [21] (rigid environment) show that the environment's stiffness has a significant influence on the transition phase behavior. In [42] it is shown that rigid body models can provide, in certain cases, better prediction than flexible ones, using appropriate nonsmooth mathematical tools [14]. Pioneering fundamental work in the field of complete robotic task control can be found in the work of Mills and Lokhorst [16], who considered the control of $n$-dof (degree-of-freedom) rigid manipulators evolving either in a free-space or in contact with a compliant environment. A simple switching controller is considered which basically consists of a motion control law and a force/position control law applied when contact is established or not. Roughly speaking, the open-loop as well as the closed-loop systems are composed of two smooth vector-fields. The stability analysis consists of the application of Lyapunov stability concepts for simple hybrid dynamical systems [2], i.e., one requires the existence of a unique positive definite function $V$ such that $\dot{V}$ is negative definite along both vector-field trajectories. This is obtained via suitable feedback-gain choices. Existence of solutions at the switching times is carefully analyzed in [16] via concepts related to differential inclusions (since at that time the control input is not uniquely defined). Other related works can be found in [6], [17], [18], [24], [45], [50], [51], [57], [58], [62], and [63]. In particular, in [63], a complementary slackness model is formulated for systems with inequality constraints (but simple transition phase), and the existence of solutions results is developed. It is noteworthy, however, that the systems considered in [16] do not belong to the class of systems subject to unilateral constraints, due to the environment's compliance. Therefore, this result cannot be considered as an extension of the work on constrained manipulators [5], i.e., control of 
systems represented by the following set of equations:

$$
\begin{aligned}
M(q) \ddot{q}+C(q, \dot{q}) \dot{q}+g(q) & =U \\
F(q) & \geq 0 \\
\text { Law (percussion, velocity) } & =\text { true }
\end{aligned}
$$

where (1) is the dynamical equation of rigid manipulators in free-space, $q \in \mathbb{R}^{n}$ is a vector of generalized coordinates, $M(q) \in \mathbb{R}^{n \times n}$ is the positive-definite inertia matrix, $C(q, \dot{q}) \dot{q}$ contains Coriolis and centrifugal acceleration terms, $g(q) \in$ $\mathbb{R}^{n \times 1}$ is the vector of generalized gravity torques, and $U \in$ $\mathbb{R}^{n \times 1}$ is the control input vector. More generally, (1) may represent the dynamics of any Lagrangian system, provided one knows stabilizing controllers for free-motion, constrainedmotion, and transition phases. Mechanisms with clearances do not, in general, belong to such a class of systems. Inequality (2) represents the set of unilateral constraints, i.e., the subspace $\Phi \subset \mathbb{R}^{n}$ within which the system evolves. Equation (3) is a physical law that relates the interaction between the robot's tip and the surface $F(q)=0$, and the generalized velocity $\dot{q}$, when contact is made at $t=t_{k}$, i.e., $F\left[q\left(t_{k}-\delta\right)\right]>0$ for any small enough $\delta>0$ and $F\left[q\left(t_{k}\right)\right]=0$.

In this paper, we shall consider the control of dynamical systems as in (1)-(3) with $F(q) \in \mathbb{R}^{m}$, i.e., we model the contact process from a rigid body point of view. We restrict ourselves to codimension-one surfaces of constraint ( $m=1$ ) or to hypersurfaces $F_{i}(q)=0, i \in \mathcal{I}$, which are mutually orthogonal with respect to the kinetic metric defined as $\langle x y\rangle=x^{T} M(q) y, x, y \in \mathbb{R}^{n}$. Here $\mathcal{I} \subset\{1, \cdots, m\}$ represents the set of constraints which are attained at the same time. The orthogonality condition is expressed as

$$
\nabla_{q} F_{i}(q)^{T} M^{-1}(q) \nabla_{q} F_{j}(q)=0
$$

$i, j \in \mathcal{I}, i \neq j$. We assume that the hypersurfaces $F_{i}(q)=$ $0, i=1, \cdots, m$ are frictionless. The reasons for these assumptions will appear clearly in the next sections and are related to the wellposedness of the collision model in (3), the existence of solutions, and the stabilization of the system in (1)-(3).

In particular, the existence of solutions for such a dynamical system is a hard problem. It has been proved in some particular cases, limited to codimension-one frictionless constraints; see, e.g., [9] and [26] or with friction [15]. Uniqueness also has been proved in [25] and [26] under some restrictions on the constraint and on the external action as well as for the lossless case only. Other studies on existence and uniqueness from the linear complementarity problem point of view can be found in [60] and [61]. In this paper we shall content ourselves with existence results, disregarding uniqueness problems. This choice is done mainly because of the great difficulty in proving such results for general impacting systems (and such studies are clearly outside the scope of this work), and also since there are no uniqueness results available in the mathematical literature for the dissipative shocks case, which is of main interest for practical control purposes.

In summary, contrary to the constrained case where one can assume that the system evolves permanently on a submanifold $F(q)=0$ with $F: \mathbb{R}^{n} \rightarrow \mathbb{R}^{m}, m \geq 1$, things are not so simple when the transition from free to constrained motion is taken into account. Although we do not claim that the case of several unilateral constraints should be disregarded (it does, on the contrary, represent a challenge in impact dynamics to define impact rules for such cases [12], [14]), we prefer in this paper to restrict ourselves to the above cases. The results were outlined in [4].

The paper is organized as follows: in Section II we introduce a possible way of modeling the system in (1)-(3) and the corresponding definitions of solutions. In Section III, some stability concepts related to the particular dynamics involved are presented. We treat in detail a one-dof case, and we analyze some stability properties for various control strategies in Section IV. In Section V, the extension to $n$-dof rigid manipulators is analyzed. Conclusions are given in Section VI. Some mathematical tools used in the paper are given in the Appendix.

\section{DyNAMic Model}

In this section, we provide some explanations and details concerning the dynamic model that will be used throughout the paper for control design purposes. We also give the definition of the trajectories using a result given in [9].

\section{A. Impact Dynamics}

1) Codimension-One Constraint: Before writing the whole set of equations that model the system, let us recall the basic facts concerning impact dynamics. Further information can be found, for instance, in [8] and [14]. As long as the configuration $q \in \mathbb{R}^{n}$ is such that $F(q)>0$, then the system's trajectories $q(t), \dot{q}(t)$ are well defined as absolutely continuous-time functions, solutions of the ODE in (1) (where $U$ is assumed to satisfy some classical regularity conditions). The same conclusions hold when $F(q) \equiv 0$ on a nonzero time interval. Now assume that for some $t=t_{k}, F[q(t)]>$ 0 for $t \in\left[t_{k}-\delta, t_{k}\right)$ and some $\delta>0$ small enough, and $F\left[q\left(t_{k}\right)\right]=0$. Assume also that $\dot{q}\left(t_{k}^{-}\right)^{T} \nabla_{q} F\left[q\left(t_{k}\right)\right]<$ 0 where $\dot{q}\left(t_{k}^{-}\right)=\lim _{t \rightarrow t_{k}, t<t_{k}} \dot{q}(t)$ and $\nabla_{q} F\left[q\left(t_{k}\right)\right]$ is the gradient of $F(q)$ at $t=t_{k}$ (that is supposed to be different from zero in the region of interest). This condition means that the velocity points outwards from the domain $\Phi$ at the point $q\left(t_{k}\right) \in \partial \Phi$. Then, a collision at $q\left(t_{k}\right)$ occurs, and the velocity $\dot{q}(t)$ possesses a discontinuity at $t=t_{k}$ such that $\dot{q}\left(t_{k}^{+}\right)^{T} \nabla_{q} F\left[q\left(t_{k}\right)\right] \geq 0$, i.e., the right-limit $\dot{q}\left(t_{k}^{+}\right)=$ $\lim _{t \rightarrow t_{k}, t>t_{k}} \dot{q}(t)$ of the velocity points inwards $\Phi$. Notice that the jump in $\dot{q}$, denoted as $\sigma_{\dot{q}}\left(t_{k}\right)$, has to be specified through a so-called restitution rule which relates post- and preimpact velocities. We denote it for the moment as

$$
\dot{q}\left(t_{k}^{+}\right)=R\left[\dot{q}\left(t_{k}^{-}\right)\right] .
$$

Remark 1: The fact that a jump in the velocity is necessary when the surface $F(q)=0$ is attained in a nontangential manner and that the impact rule must be such that the postimpact velocity points inwards $\Phi$, renders the domain $\Phi$ invariant under the dynamics. The nonsmooth impact theory developed in [14] and [15] precisely aims at studying a general model that makes $\Phi$ such a domain. 
Now let us note that since (5) implies a discontinuity in the velocity at $t=t_{k}$, it follows that at $t=t_{k}$ there must be a percussion $P_{q}\left(t_{k}\right)$ acting on the system, i.e., a generalized impulsive force of the form $P_{q}\left(t_{k}\right) \delta_{t_{k}}$, where $\delta_{t_{k}}$ is the Dirac measure at $t_{k}$. Also at $t_{k}$ the acceleration is given by

$$
\ddot{q}=\sigma_{\dot{q}}\left(t_{k}\right) \delta_{t_{k}}
$$

where ${ }^{1} \sigma_{\dot{q}}\left(t_{k}\right)=\dot{q}\left(t_{k}^{+}\right)-\dot{q}\left(t_{k}^{-}\right)$. It follows that systems as in (1)-(3) are represented by measure differential equations, i.e., differential equations containing singular distributions (like the Dirac measure) in their right-hand side [34]. It is then possible to prove (see, e.g., [64]) that at $t=t_{k}$ the dynamical equations become

$$
M\left[q\left(t_{k}\right)\right] \sigma_{\dot{q}}\left(t_{k}\right)=P_{q}\left(t_{k}\right) .
$$

It is also important to note that $q(t)$ is continuous at impact times; see [64]. Equations (5) and (7) make the impact problem complete, in the sense that given preimpact conditions $\left[q\left(t_{k}\right), \dot{q}\left(t_{k}^{-}\right)\right]$one is able to calculate both $\dot{q}\left(t_{k}^{+}\right)$[from (5)] and the percussion vector [from (7)].

Let us now focus on the restitution rule in (5). In the onedimensional case, the most widely used rule is known as Newton's rule, which states that if a particle strikes a rigid obstacle; then

$$
\dot{q}\left(t_{k}^{+}\right)=-e \dot{q}\left(t_{k}^{-}\right)
$$

where $e \in[0,1]$ is the restitution coefficient. In higherdimensional cases, we shall apply the rule [12], [13]

$$
\dot{q}\left(t_{k}^{+}\right)^{T} \nabla_{q} F\left[q\left(t_{k}\right)\right]=-e \dot{q}\left(t_{k}^{-}\right)^{T} \nabla_{q} F\left[q\left(t_{k}\right)\right]
$$

which can be seen as a generalized normal rule (only one coefficient is needed in (9) since $\dot{q}^{T} \nabla_{q} F(q)$ is scalar). The remaining part of the velocity can be computed from (7). Indeed there are $(n+1)$ unknown parameters to the problem (the $n$-velocity components $\dot{q}\left(t_{k}^{+}\right)$and the unique component of $P_{q}$ which verifies $P_{q}=p_{q} \nabla_{q} F(q)$ for some $p_{q} \in \mathbb{R}^{+}[14]$ ), and (7) and (9) provide us with $(n+1)$ equations. We shall come back later on the calculations of postimpact velocities using some particular coordinates such as the ones proposed in [5].

2) Constraints of Codimension $\geq 2$ : In the case $F(q) \in$ $\mathbb{I R}^{m}, m \geq 2$, there may be several hypersurfaces attained at the same time. At such singular points, $\partial \Phi$ is not smooth so that it is really given by the intersection of several hypersurfaces. The question is: can the restitution rules in (8) and (9) be generalized to such cases? Does this generalization yield a coherent result? The answer is yes, if the hypersurfaces that form the singularity satisfy (4); see [12] and [13, Th. 2, pp. 26 and 27]. This fact is clearly explained as follows. Let us endow each surface $F_{i}(q)=0, i \in \mathcal{I}$ with the kinetic metric defined as above. Then the normal unit vector to $F_{i}(q)=0$ is given by [27]

$$
n_{q, i}=\frac{M^{-1}(q) \nabla_{q} F_{i}(q)}{\sqrt{\nabla_{q} F_{i}(q)^{T} M^{-1}(q) \nabla_{q} F_{i}(q)}} .
$$

\footnotetext{
${ }^{1} \sigma_{f}\left(t_{k}\right)$ will be used generically to denote the jump in the function $f(t)$ at time $t_{k}$.
}

Define $(n-2)$ vectors $t_{q, i} \in \mathbb{R}^{n}$ such that $t_{q, i}^{T} M(q) t_{q, j}=0$ for $i \neq j, t_{q, i}^{T} M(q) n_{q, j}=0$, and $t_{q, i}^{T} M(q) t_{q, i}=1$. Then the basis $\left(n_{q, 1}, n_{q, 2}, t_{q, 1}, \cdots, t_{q, n-2}\right)$ is orthonormal; see (4). Define now the generalized velocity transformation

$$
\left[\begin{array}{c}
\dot{q}_{\text {norm }} \\
\dot{q}_{\text {tang }}
\end{array}\right]=\left[\begin{array}{c}
n_{q}^{T} \\
t_{q}^{q}
\end{array}\right] M(q) \dot{q}
$$

where the matrices $n_{q} \in \mathbb{R}^{2 \times n}$ and $t_{q} \in \mathbb{R}^{(n-2) \times n}$ are defined from the above vectors. The coordinates $\dot{q}_{\text {norm }}$ and $\dot{q}_{\text {tang }}$ in the frame $\left(n_{q, 1}, n_{q, 2}, t_{q, 1}, \cdots, t_{q, n-2}\right)$ represent the projections of $\dot{q}$ on $n_{q, 1}, n_{q, 2}, t_{q, 1}, \cdots, t_{q, n-2}$, respectively, in the sense of the kinetic metric. Recall that the percussion vector $P_{q}$ verifies $P_{q}=p_{q, 1} \nabla_{q} F_{1}(q)+p_{q, 2} \nabla_{q} F_{2}(q)$ for some $p_{q, 1}, p_{q, 2} \in \mathbb{R}^{+}$. Then it is not difficult to show that the dynamical equations at the impact time are given by

$$
\left\{\begin{aligned}
\sigma_{\dot{q}_{\mathrm{norm}, 1}} & =n_{q, 1}^{T} P_{q} \\
\sigma_{\dot{q}_{\mathrm{norm}, 2}} & =n_{q, 2}^{T} P_{q} \\
\sigma_{\dot{q}_{\mathrm{tang}}} & =t_{q}^{T} P_{q}=0
\end{aligned}\right.
$$

which is another way to write (7). (If $F(q) \in \mathbb{R}$, then there is only one component to $\dot{q}_{\text {norm }}$.) Now note that if (4) is satisfied for $i=1, j=2$, then (12) becomes

$$
\left\{\begin{aligned}
\sigma_{\dot{q}_{\mathrm{norm}, 1}} & =p_{q, 1} n_{q, 1}^{T} \nabla_{q} F_{1}(q) \\
\sigma_{\dot{q}_{\mathrm{norm}, 2}} & =p_{q, 2} n_{q, 2}^{T} \nabla_{q} F_{2}(q) \\
\sigma_{\dot{\dot{q}_{\mathrm{tang}}}} & =0
\end{aligned}\right.
$$

i.e., the percussion component $p_{q, 1}$ has no influence on the velocity $\dot{q}_{\text {norm, }}$ jump, and vice-versa. It is then possible and coherent to define a restitution rule [which is a generalization of that in (9)] as

$$
\left\{\begin{array}{l}
\dot{q}_{\text {norm, } 1}\left(t_{k}^{+}\right)=-e_{1} \dot{q}_{\text {norm, }, 1}\left(t_{k}^{-}\right) \\
\dot{q}_{\text {norm, } 2}\left(t_{k}^{-}\right)=-e_{2} \dot{q}_{\text {norm, } 2}\left(t_{k}^{-}\right) .
\end{array}\right.
$$

Note that from the definition of these quantities, we have

$$
\begin{aligned}
& \dot{q}\left(t_{k}^{+}\right)^{T} \nabla_{q} F_{1}(q)=-e_{1} \dot{q}\left(t_{k}^{-}\right)^{T} \nabla_{q} F_{1}(q) \\
& \dot{q}\left(t_{k}^{+}\right)^{T} \nabla_{q} F_{2}(q)=-e_{2} \dot{q}\left(t_{k}^{-}\right)^{T} \nabla_{q} F_{2}(q) .
\end{aligned}
$$

The incoherence when (4) is not verified comes from several facts. It is shown in [12] via a sequence of approximating compliant problems (the surfaces of constraint are replaced by a spring + damper model) that there is no hope of any sort of convergence toward the rigid limit case. Continuity of the trajectories with respect to the initial conditions is guaranteed if orthogonality is satisfied [13, Th. 2, pp. 26 and 27]. This last fact is easily seen on a planar example of a particle striking in an angle. Notice that we do not claim that the restitution rule as defined in (11)-(16) is the only possible model of collision one might choose. Moreover, it is well known that Newton's conjecture about the restitution law has to be verified in experiments like any other model. For instance, it is known that $e$ may depend on $\dot{q}_{\text {norm }}\left(t_{k}^{-}\right)$ [8]; see also [43] for experimental results on a falling rod, which show that micro-collision effects play a crucial role in the impact phenomenon. This is even more true in the case of $n$-dimensional articulated systems such as manipulators. 
In view of the studies available in the mechanical literature [12]-[14], Newton's conjecture in its generalized form seems to be the most reasonable model concerning multiple rigidbody impacts. It has also been confirmed experimentally for colliding manipulators; see e.g., [21] and [56], although much more experimental validations are needed. It is finally a theoretically sound model, as we discuss in Section II-C, which is a necessary first step in the analysis; the stability analysis framework developed in this paper can accommodate more elaborated impact models.

We are now able to propose a general model for the system described in (1)-(3).

\section{B. A General Form of the Dynamical System}

From the above discussions, it is reasonable and natural to split the dynamical equations into three parts as follows (this is written for a codimension-one constraint and can be easily generalized to the case $m \geq 2$, provided the above conditions are satisfied).

Free-Motion Phases:

$$
\begin{aligned}
M(q) \ddot{q}+C(q, \dot{q}) \dot{q}+g(q) & =U \\
F(q) & \geq 0 .
\end{aligned}
$$

Constrained-Motion Phases:

$$
\begin{aligned}
M(q) \ddot{q}+C(q, \dot{q}) \dot{q}+g(q) & =U+\lambda \frac{\partial F}{\partial q}(q) \\
F(q) & =0 \text { and } \lambda \geq 0 .
\end{aligned}
$$

Transition Phases:

$$
\begin{aligned}
M(q) \ddot{q}+C(q, \dot{q}) \dot{q}+g(q) & =U \\
F(q) & >0 \\
M\left[q\left(t_{k}\right)\right] \sigma_{\dot{q}}\left(t_{k}\right) & =P_{q}\left(t_{k}\right) \\
F\left[q\left(t_{k}\right)\right] & =0 \\
\dot{q}\left(t_{k}^{+}\right)^{T} \nabla_{q} F\left[q\left(t_{k}\right)\right] & =-e \dot{q}\left(t_{k}^{-}\right)^{T} \nabla_{q} F\left[q\left(t_{k}\right)\right] .
\end{aligned}
$$

Notice that once the transition phase is finished \{i.e., $\left.\dot{q}\left(t_{k}^{+}\right)^{T} \nabla_{q} F\left[q\left(t_{k}\right)\right]=0\right\}$, two situations can occur: either the applied forces are such that the system remains in contact with the boundary of $\Phi$, or it detaches. This depends on the Lagrange multiplier $\lambda$ sign: if $\lambda \geq 0$, constrained motion is insured; if $\lambda<0$, the system detaches from the constraint surface. In a case as in (28), this can be written as follows [12]: $\ddot{q}=\max [0, U]$ if $\dot{q}\left(t_{k}^{+}\right)=0, q\left(t_{k}\right)=0$ since the multiplier $\lambda=-U .^{2}$ In our case such outcomes are a consequence of the controller $U$. Since we do not suppose a priori that a unique controller $U_{i}$ is applied, the complete dynamical system is given by the above three phases plus a control strategy of the following form.

\section{Control Strategy:}

$$
\begin{aligned}
& U_{i} \in \mathcal{U} \\
& U=U_{i} \quad \text { if condition } C_{i} \text { is true. }
\end{aligned}
$$

\footnotetext{
${ }^{2}$ Such transitions may be taken care of by so-called complementarity conditions; see, e.g., [60].
}

$\mathcal{U}$ is a set of controllers which stabilizes the system when it evolves in one of the three phases mentioned above. ${ }^{3}$ Hence, $\mathcal{U}$ contains position as well as force/position controllers. The condition $C_{i}$ may be seen as a high-level controller, possibly with an associated automaton [3]. They may be event-based or open loop. One must, however, take care of existential problems to the overall dynamical system. As we shall see later, it is also possible to interpret $U$ as a unique controller, discontinuous in $t$ and/or in the system's state (depending on the nature of the conditions $C_{i}$ ).

Remark 2: The equation in (20) represents the smooth dynamics between the impact times, i.e., on intervals $\left(t_{k}, t_{k+1}\right)$. Furthermore, notice that in general the closed-loop state variables contain the position tracking error $\tilde{q}=q(t)-q_{d}(t)$, where $q_{d}(t) \in C^{2}\left[\mathbb{R}^{+}\right]$represents some desired motion. Then the unilateral constraint $F(q) \geq 0$ can be written as $F_{t}(\tilde{q}) \triangleq F\left(\tilde{q}+q_{d}\right) \geq 0$. In other words, the invariant constraint can be written in closed-loop form as a time-varying constraint. The restitution rule in (9) then can be rewritten as

$$
\nabla_{\tilde{q}} F_{t}^{T} \dot{\tilde{q}}\left(t_{k}^{+}\right)=-e \nabla_{\tilde{q}} F_{t}^{T} \dot{\tilde{q}}\left(t_{k}^{-}\right)-(1+e) \nabla_{t} F_{t}
$$

Roughly speaking, since the constraint is time-varying, the restitution rule must incorporate its velocity at the impact time. This is represented in the last term of the right-hand side of (25). From the definition of the time-varying closed-loop unilateral constraint $F_{t}(\tilde{q})$, it is easy to verify that $\nabla_{\tilde{q}} F_{t}=$ $\nabla_{q} F$ and that $\nabla_{\tilde{q}} F_{t}^{T} \dot{q}_{d}=\nabla_{t} F_{t}$ so (9) and (25) are equivalent. This is simply to show that we could have formulated the dynamical system in (17)-(22) in terms of closed-loop (or tracking errors) variables.

Remark 3: It is clear that the aim of the robotic task is to make the manipulator track some desired trajectories. During free- and constrained-motion phases, one classically defines desired position, velocity, and interaction force trajectories. The controller $U$ has to be designed such that the tracking errors vanish asymptotically. The system's behavior during the transition phase will be, in general, quite different. Indeed, in some tasks it may be required that impact times verify $t_{k+1}=$ $t_{k}+T$ for some $T>0$ with bounded trajectories on $\left(t_{k}, t_{k+1}\right)$ (one can think of a "hammer"-like task). This also implies that $U$ in (20) is designed such that the sequence of impact times $\left\{t_{k}\right\}$ does exist. Note that in general the controller stabilizing free-motion phases will not be suitable for the transition phases' objectives. In this paper, we will consider only robotic tasks which involve alternatively free- and constrained motion phases. Although the model in (17)-(24) might represent other classes of mechanical systems (for instance, bipedal robots and control of such systems [41] or simple impacting systems with chaotic dynamics like the bouncing ball or the impact oscillator [52]-[54]), we do not consider such extensions for the moment. Hence, the goal of the controller during the transition phases is to guarantee that the manipulator stabilizes on the constraint surface $F(q)=0$ in finite time. In other words we shall require that the sequence $\left\{t_{k}\right\}$ has a finite accumulation point $t_{\infty}<+\infty$ with $F\left[q\left(t_{\infty}\right)\right]=0$. Except in

\footnotetext{
${ }^{3}$ One of the main conclusions of the experimental work in [44] is that three distinct controllers have to be used for the control of a complete task.
} 
the case $e=0,\left\{t_{k}\right\}$ is an infinite sequence. $t_{\infty}<+\infty$ implies that $e<1$, i.e., there is a dissipation of energy at collisions.

Remark 4: Using the generalized coordinates transformation described in [5], the unilateral constraint $F(q) \geq 0$, $F(q) \in \mathbb{R}^{m}$ can be rewritten in a new set of coordinates $X \triangleq\left(\begin{array}{l}x_{1} \\ x_{2}\end{array}\right)$, where $x_{1} \in \mathbb{R}^{m}$ and the constraint $F(q)=$ 0 becomes simply $x_{1}=0$. This well-known transformation relies on a suitable partition of the coordinates $q$ and may be assumed for convenience to be global. The constraints $F_{i}(q)$ are also assumed to be independent. Therefore, the constraint $F(q) \geq 0$ can be rewritten as $x_{1} \geq 0$ so that the Euclidean normal vector to the constraint hypersurface $x_{1, i}=0$ is simply the $i$ th unit vector $\varepsilon_{i} \in \mathbb{R}^{n}$. It is noteworthy that although the rest of the coordinates $x_{2} \in \mathbb{R}^{n-m}$ represent the motion of the system along the tangential part of the constraint, $\dot{x}_{2}$ does not in general remain continuous at the impact times if one adopts the generalized Newton's restitution rule described in (9)-(16). Indeed, one has to compute the jump in $\dot{x}_{1}$ from the restitution rule and then compute the jump in $\dot{x}_{2}$ from (7), i.e., the algebraic dynamical equation at the shock instant. We shall come back to these calculations in Section V-B. In fact, the velocity transformation in (11) can be performed starting from any set of generalized coordinates, in particular $X$. One concludes that for simplicity (but not without loss of generality since the transformation is assumed to hold globally) the unilateral constraints could be written as $q_{i} \geq 0,1 \leq i \leq m$. Also the restitution rule in (21) becomes $\dot{q}_{i}\left(t_{k}^{+}\right)=-e_{i} \dot{q}_{i}\left(t_{k}^{-}\right)$ when $m \geq 1$.

\section{Definition of the Solutions}

The classical bouncing ball example (see, e.g., [35]) suggests that the solution $q(t)$ of an impact problem possesses derivatives which are not piecewise continuous but rather right continuous of local bounded variation (RCLBV) in time; see, e.g., [14]. This also allows us to define the acceleration as a bounded positive measure since it is the derivative of an RCLBV function [36]. Nevertheless, in the general case of a system as in (1)-(3), such assertions are not trivial and must be proved. Results in this direction can be found in [9]. In particular, [9, Th. 2] requires that a) the system evolves in a convex domain of the state space, i.e., the region $\Phi$ defined by inequalities (2) must be convex, b) with a regular (i.e., twice differentiable) boundary $\partial \Phi$, and c) the external action $U(t)$ on the system is continuous in time. This may not be satisfied for systems like in (1)-(3). However, we have seen in Remark 4 that the coordinate change like the one proposed in [5] and a suitable feedback control law allow us to put the closedloop system in the framework developed in [9]. In particular, this allows us to get a convex domain $\Phi$. The convexity of $\Phi$ is not a real obstacle, however, as long as locally, existence and uniqueness of a projection on $\partial \Phi$ is assured. Multiplicity of the constraints (i.e., regularity of $\partial \Phi$ ) is a more serious problem. Existence has been proved only for zero energy loss at impacts [10]. Notice that this is not sufficient here because we need finite time stabilization results which cannot be obtained when $e=1$. Continuity of the external action in $t$ may also be relaxed to measurability in the Lebesgue sense [11] and with Carathéodory-like conditions [34] since the crucial point is to guarantee existence of solutions of a penalizing problem (see [9, Th. 2]). Although some dynamical problems with unilateral constraints may not possess any solution with $\dot{q}(t) \in R C L B V$, we conjecture that under the restrictions imposed on the unilateral constraints (i.e., either uniqueness or multiplicity but for orthogonal constraints), the existence is assured. Hence, in the following, we shall always assume that $\dot{q} \in R C L B V$. Furthermore, notice that this has some nice consequences for the stability analysis purposes since RCLBV functions possess a countable set of discontinuity points on any compact time interval (hence are Riemann integrable). It is, consequently, natural to associate a discrete-time system (or impact Poincaré map) to such impacting systems. This is a natural extension in the set of solutions of bounded variation of the results in [1], where only piecewise continuous solutions are considered.

Another problem appears at the switching times between the different controllers $U_{i}$; see (23). Two situations may be considered: either the switching period has a strictly positive measure, or it has a zero measure. It seems realistic to assume a zero measure, since in practice the switching will generally be obtained via software and consequently will be almost instantaneous. Hence, what happens "during" the switch will be disregarded in the stability analysis. Finally which sense should we give to the control law at the switching times? The point of view of differential inclusions is adopted in [16], i.e., $U \in\left[U_{\min }, U_{\max }\right]$ at the switching times. Then, the overall system solutions have to be considered as reachable sets, no longer time functions and existence as well as uniqueness. Stability results have to be generalized; see [34]. As noted in [16], no general mathematical tool seems available in the mathematical literature to study differential inclusions with measures in the right-hand side or with state jump conditions. It would be possible to define control strategies such that no collision occurs at the switches. We, however, prefer to assume in this study that at a switching time $T_{s}$ the controller takes a value between $U\left(T_{s}^{-}\right)$and $U\left(T_{s}^{+}\right)$, which can be unknown. Actually we formulate the problem with a time-discontinuous controller, presumed to be right continuous. This is not so elegant from a theoretical point of view but allows us to get rid of a problem with few practical consequences on the stability analysis, as long as the switching periods are supposed to be instantaneous.

\section{STABILITY ANALYSIS FRAMEWORK}

The system in (17)-(24) is a complex hybrid dynamical system [3] which involves continuous as well as discrete time phases. Stability criteria have been proposed for simple hybrid systems; see [2]. However, they do not apply to more complicated systems as in (17)-(24). In this note we choose a time-domain approach close to Lyapunov's second method. The stability analysis will therefore be based on the choice of a suitable unique autonomous positive definite function $V$ of the system's state. Although this seems the simplest and the most natural way to proceed, it is in fact not clear which conditions of variation of $V$ should be required. In general, 
the controllers may be dynamic output or state feedback laws. Hence, (17)-(24) do not represent the whole closedloop system. Note that for the moment the representation of the transition phase in (20)-(22) is not very tractable. Indeed, it is amenable for stability analysis via the tools developed in [1] which require that a positive definite function $V$ of the state be such that $\dot{V} \leq 0$ along the trajectories of (20) and $\sigma_{V}\left(t_{k}\right) \leq 0$ at the impact times. The basic idea is roughly to get simultaneously continuous and discrete stability via the same function $V$. However, as the simple bouncing ball example shows, it may not be obvious to verify such stability criterion in practical cases. Moreover, the theory developed in [1] relies on piecewise continuous solutions, i.e., the impact times verify $t_{k+1}>t_{k}+\delta$ for some $\delta>0$. Still, the bouncing ball example with $e \in(0,1)$ proves that this is not sufficient to study impacting systems in general. More precisely, the stability analysis framework proposed in [1] is well-suited when one wants to stabilize the manipulator close to the obstacle; see [64, ch. 7] for more details. In this case accidental collisions may occur between the robot's tip and the constraint surface. Such a stability criterion guarantees that these collisions do not destroy the Lyapunov stability of the closed-loop system. For a system submitted to unilateral constraints, this corresponds to $x^{\star} \in \operatorname{Int}(\mathcal{D})$, where $\mathcal{D}=$ $\Phi \times \mathbb{R}^{n} \ni(q, \dot{q})$. The dynamical equations are then given by (17) and (18). The inequality in (18) allows the collisions to occur even during free-motion phases. Notice that due to the form of the unilateral constraints in (2), $\Phi$ is closed. In a robotic task, we may be interested in having stabilization on the constraint, i.e., $x^{\star} \in \partial \Phi \times \mathbb{R}^{n}$. Roughly speaking, classical stability concepts on metric spaces [33] do no carry out to this situation, where it is not possible to define neighborhoods (open domains) of $x^{\star}$ on $\partial \Phi \times \mathbb{R}^{n} \cdot{ }^{4}$ A way to overcome this problem for the analysis of the transition phase is to study the impact Poincaré map ${ }^{5}$ associated with (20)-(22). In general, such maps are difficult to obtain explicitly. However, one may use the control input $U$ in (20) to simplify the smooth dynamics (for instance feedback linearization) and make it possible to get explicitly the discrete-time system associated with (20)-(22). This path will be investigated in details in the sequel, where indeed the goal will be to find a controller $U$ in (20) such that (20)-(22) define a stable discrete-time operator. Another way to avoid this problem is to define nonsmooth changes of coordinates which transform systems with unilateral constraints as in (1)-(3) into systems with time-continuous trajectories [38].

To clarify the proposed stability criterion, and to overcome those difficulties, we introduce the following definitions. Let us split the time axis into intervals $\Omega_{k}$ and $I_{k}$, corresponding to smooth phases [during which collisions may nevertheless

\footnotetext{
${ }^{4}$ The authors are in fact aware of only one reference dealing with Lyapunov stability of such critical fixed points when the forces acting on the system are zero (i.e., $\lambda=0$ ); see [37]. This case is not very interesting, in general, since one wants to apply a nonzero force on the environment.

${ }^{5}$ With some abuse of name since Poincaré maps are defined (strictly speaking) from periodic trajectories [27], whereas here it will concern solutions converging to zero in finite time (but with an infinity of rebounds).
}

occur at times $t=t_{k}{ }^{6}$ for motion phases in (17), but the closedloop equations fixed-point belongs to $\operatorname{Int}(\Phi)]$ and transition phases, respectively (the goal is to obtain stabilization of the system on the surface $\partial \Phi)$. From the hybrid dynamical systems point of view, the $\Omega_{k}$ 's correspond to the continuous-time phases and the $I_{k}$ 's to discrete-time phases. As we shall see, $I_{k}$ does not necessarily correspond to the rebounds phase, but more generally contains it. Even in the case of plastic impact $(e=0)$, in general $\lambda\left[I_{k}\right]>0(\lambda[\cdot]$ is the Lebesgue measure). Note that $\mathbb{R}^{+}=\bigcup_{k} \Omega_{k} \bigcup_{k} I_{k}$. The phases $\Omega_{k}$ and $I_{k}$ may be finite or infinite. We denote $\Omega_{k}=\left[T_{0}^{k}, T_{f}^{k}\right]$ and $I_{k}=\left[t_{0}^{k}, t_{f}^{k}\right]$. Note that $\Omega_{k}$ corresponds to free- as well as constrained motion phases. Hence there cannot be more than two sequential intervals $\Omega_{k}, \Omega_{k+1}$, since the transition from free- to constrained motion phases must be an impact phase. Hence we have for a typical task

$$
\begin{aligned}
\mathbb{R}^{+}= & \Omega_{0} \cup I_{0} \cup \Omega_{1} \cup \Omega_{2} \cup I_{1} \\
& \cup \cdots \Omega_{2 k-1} \cup \Omega_{2 k} \cup I_{k} \cdots .
\end{aligned}
$$

Let us define $\Omega$ as the complement of $I=\cup_{k} I_{k}$ so that $\lambda[\Omega]=$ $+\infty$. We now introduce the proposed stability framework. In the following, $x(t)$ denotes the state of the closed-loop system (17)-(24), with $x(t) \in R C L B V . V(x)$ denotes a positive definite function, continuous in $x$, satisfying $\alpha(\|x\|) \leq V(x) \leq$ $\beta(\|x\|)$, where $\alpha($.$) and \beta($.$) are class K$ functions. ${ }^{7}$ Also $\gamma(\cdot)$ and $\gamma_{1}(\cdot), \gamma_{2}(\cdot)$ are class $K$ functions. Notice that $x(t) \in \mathbb{R}^{l}$, $l \geq 2 n$ may be different from $(\tilde{q}, \dot{\tilde{q}})$, mainly because of dynamic state feedback controllers. Let us finally define a particular decomposition of $x$ as $x^{T}=\left[\begin{array}{lll}\xi_{1}^{T} & \xi_{2}^{T}\end{array}\right], \xi_{1} \in \mathbb{R}^{n_{1}}$, $\xi_{2} \in \mathbb{R}^{n_{2}}$. Define $\Omega^{1}$ and $\Omega^{2}$ such that $\lambda\left[\Omega^{1}\right]=+\infty$, $\lambda\left[\Omega^{2}\right]=+\infty$, and $\Omega=\Omega^{1} \cup \Omega^{2}$.

Lemma 1-Weak Stability: Assume that on intervals $I_{k}$, $V\left(t_{f}^{k}\right) \leq V\left(t_{0}^{k}\right)$ along the system's trajectories, $V$ is uniformly bounded on $I_{k}$, and $\lambda\left[I_{k}\right]<+\infty$ for all $k \geq 0$. Then the following assertions hold.

1) If on $\Omega, \dot{V}[x(t)] \leq-\gamma[\|x(t)\|]$ for $t \neq t_{k}$ along system's trajectories, and $\sigma_{V}\left(t_{k}\right) \leq 0$, then $x(t) \rightarrow 0$ as $t \rightarrow+\infty, t \in \Omega$.

2) If $\dot{V}[x(t)] \leq-\gamma_{1}\left[\left\|\xi_{1}(t)\right\|\right]$ for $t \neq t_{k}$ on $\Omega^{1}, \dot{V}[x(t)] \leq$ $-\gamma_{2}\left[\left\|\xi_{2}(t)\right\|\right]$ for $t \neq t_{k}$ on $\Omega^{2}, \sigma_{V}\left(t_{k}\right) \leq 0$ for $t_{k} \in \Omega$, $\left\|\xi_{1}\right\|$ nonincreasing on $\Omega^{2},\left\|\xi_{2}\right\|$ nonincreasing on $\Omega^{1}$, then $x(t) \rightarrow 0$ as $t \rightarrow+\infty, t \in \Omega$.

3) If $\lambda\left[\Omega^{1}\right]<+\infty, \lambda\left[\Omega^{2}\right]=+\infty, \lambda[I]<+\infty, \xi_{1}(t)$ is constant on $\Omega^{2}, V\left(\nu, \xi_{2}\right)-V(\nu, 0) \geq 0$ for any $\nu \in \mathbb{R}^{n_{1}}$, and $\dot{V}[x(t)] \leq-\gamma_{2}\left[\left\|\xi_{2}(t)\right\|\right]$ for $t \neq t_{k}$, $\sigma_{V}\left(t_{k}\right) \leq 0$, then $\xi_{2}(t) \rightarrow 0$ as $t \rightarrow+\infty$.

The proof is given in the Appendix. Note that since $V(x)$ is continuous in $x$, then $V(t) \in R C L B V$. It is thus justified to use the jumps of $V$ at the impact times in the stability analysis. Recall also that at such times $t_{k}$, the time derivative of $V(t)$ is calculated via a generalized chain rule [36], i.e., $\dot{V}\left(t_{k}\right)=$

\footnotetext{
${ }^{6}$ Throughout the paper, the impact times are generically denoted as $t_{k}$. This does not mean that a time $t_{k}$ is related to the domain $\Omega_{k}$. The subscript $k$ is a dummy variable associated to the different quantities that we need to define, i.e., the different time intervals, their upper and lower bounds, the impact times, etc. Moreover to simplify the notations, we employ $t_{k}$ for all the impact phases.

${ }^{7}$ i.e., $\alpha(0)=\beta(0)=0$; they are strictly increasing and radially unbounded.
} 
$\sigma_{V}\left(t_{k}\right) \delta_{t_{k}}$. Also, strictly speaking on $\left(t_{k}, t_{k+1}\right)$, the derivative $\dot{V}$ denotes the upper right Dini derivative of $V$ along closedloop trajectories (see, e.g., [1, Definition 13.2]). Let us remark that $\xi_{1}$ and $\xi_{2}$ may correspond to various decompositions of $x$ such as the one obtained via the McClamroch-Wang's transformation [5] described above. This will be made clear in the following sections.

Let us now define the transformations $G_{i}: x \mapsto \bar{x}_{i}^{T} \triangleq$ $\left[F_{i}(q), q_{2}, \cdots, q_{n}, \dot{q}^{T}\left(\partial F_{i} / \partial q\right), \dot{q}_{2}, \cdots, \dot{q}_{n}, z\right]^{T}$. The signal $z$ is due to a possible dynamic state feedback controller. We assume that those mappings are global diffeomorphisms so that the function $V_{i}\left(\bar{x}_{i}\right) \triangleq V \circ G_{i}^{-1}\left(\bar{x}_{i}\right)=V(x)$ is defined globally. ${ }^{8}$ The closed-loop impact Poincaré maps are defined as ${ }^{9}$

$$
\begin{aligned}
P_{\Sigma, i}: \quad \Sigma_{i} & \rightarrow \Sigma_{i} \\
\bar{x}_{\Sigma, i}(k) & \mapsto \bar{x}_{\Sigma, i}(k+1)
\end{aligned}
$$

$P_{\Sigma, i}(0)=0$, where $\Sigma_{i}$ is the impact Poincare section defined as $\Sigma_{i}=\left\{x: F_{i}(q)=0, \dot{q}^{T} \nabla_{q} F_{i}>0\right\}$. Therefore $P_{\Sigma}$ is the discrete-time operator associated with (20)-(22). In other words, the impact Poincaré map is defined from the mapping $P: x\left(t_{k}^{+}\right) \mapsto x\left(t_{k+1}^{+}\right)$as $P_{\Sigma, i}\left[\bar{x}_{\Sigma, i}(k)\right]=P$ 。 $G_{i}^{-1}\left[\bar{x}_{i}\left(t_{k}^{+}\right)\right]$, taking the restriction to $\Sigma_{i}$ of $P \circ G_{i}^{-1}$. Finally, the function $V_{\Sigma, i}$ is defined as the restriction of $V_{i}\left(\bar{x}_{i}\right)$ to $\Sigma_{i}$, i.e., $V_{\Sigma, i}\left[\bar{x}_{\Sigma, i}(k)\right]=V_{i}\left[\bar{x}_{i}\left(t_{k}^{+}\right)\right]$. In the next Lemma we introduce stronger conditions for the behavior of the system during the transition phases $I_{k}$.

Lemma 2-Strong Stability: Let $x(t)$ and $V(x)$ satisfy the conditions in a), b), or c) in Lemma 1 . Assume furthermore that on $I_{k}$, one has $q_{d} \equiv 0$ and:

i) $\sigma_{V}\left(t_{k}\right) \leq 0$

ii) $V\left(t_{k+1}^{-}\right) \leq V\left(t_{k}^{+}\right)$;

iii) $V$ is uniformly bounded and time continuous on $I_{k}$ $\cup_{k}\left\{t_{k}\right\}$, where the sequence $\left\{t_{k}\right\}$ exists and has a finite accumulation point.

Then the system is weakly stable, and the closed-loop impact Poincaré map $P_{\Sigma, i}$ is Lyapunov stable with the Lyapunov function $V_{\Sigma, i}$.

The proof follows from Lemma 1, [39], and using the fact that $V_{\Sigma, i}(k)=V\left[x\left(t_{k}^{+}\right)\right]$. Notice that the condition $V\left(t_{f}^{k}\right) \leq V\left(t_{0}^{k}\right)$ can be assured if for instance $V\left(t_{0}\right) \leq V\left(t_{0}^{k}\right)$ and condition ii) is satisfied. Although $V$ is designed as a continuous-time function, the above conditions assure that $V$ qualifies as a discrete-time Lyapunov function for the mapping $P_{\Sigma, i}$, after an eventual state-space transformation. The interest for the strong stability concept is that if conditions i)-iii) are satisfied, then the whole stability proof is led with a unique $V$. Also Lemma 2 conditions reinforce the behavior of the trajectories during the impact phase. ${ }^{10}$ Note that if the $G_{i}$ 's are equal to the identity mapping, then $V_{i}=V$, and it is sufficient that $q_{d, j} \equiv 0$ for all $1 \leq j \leq m$.

\footnotetext{
${ }^{8}$ Clearly this may imply a reordering of the coordinates before performing the transformation $G_{i}$.

${ }^{9}$ The preimpact values can be chosen as well to define this mapping.

${ }^{10}$ Notice the fact that $t_{\infty}<+\infty$ is not a consequence of the stability of $P_{\Sigma, i}$. It has to be proved.
}

The various stability criteria in Lemma 1 are meant to apply to different classes of robotic tasks. It is also possible that other criteria can be invented to cope with other situations. We, however, limit ourselves in this study to these three: a) is the most direct extension of Lyapunov second method. It can represent the case when the task is composed of free-motion phases, separated by some impact phases; case b) relaxes the conditions and is typical for tasks involving an infinity of transition phases, and where the controllers are dynamic state feedback laws; case c) may be suitable when the task ends in a smooth phase (free or constraint motion), i.e., $\lambda[I]<+\infty$.

\section{A ONE-DOF EXAMPLE}

In the following, we first illustrate on a one-dof example how the control strategy (which encompasses the switching times that define the subtasks as well as the desired trajectory definition) can influence the type of stability of the closed-loop scheme. We gradually introduce a weakly stable scheme and then show how strong stability can be obtained by modifying both the switching times and the free-motion desired trajectory. The goal of this section is mainly to show how the control strategy can be modified in various ways to comply with the stability requirements of Lemmas 1 and 2 .

\section{A. Weakly Stable Task Example}

Let us consider a simple one-dof example, i.e., a mass $m$ acted upon by a control force $U \in \mathbb{R}$ and restricted to move on the half-line $q \in[0,+\infty)$. The dynamical equations are given by

$$
m \ddot{q}=U, \quad q \geq 0 .
$$

Let us consider the control input

$$
U(t, q, \dot{q})=\alpha(t) U_{n c}(t, q, \dot{q})+[1-\alpha(t)] U_{t}
$$

where

$$
\begin{aligned}
U_{n c}(t, q, \dot{q}) & =m \ddot{q}_{d}-\lambda_{1} \dot{\tilde{q}}-\lambda_{2} \tilde{q} \\
U_{t} & =-F_{d}, \quad F_{d}>0 \\
\alpha(t) & =\left\{\begin{array}{ll}
1 & \text { for } t \in \Omega_{2 k} \\
0 & \text { for } t \in I_{k} \cup \Omega_{2 k+1}
\end{array}, \quad k \geq 0\right.
\end{aligned}
$$

and the system is supposed to be initialized with $q(0)>0$, i.e., $0 \in \Omega_{0}$. Hence $\Omega_{2 k}$ denotes free-motion phases and $\Omega_{2 k+1}$ constrained-motion phases. The free-motion desired trajectory $q_{d}(t)$ is defined as follows and is depicted in Fig. 1:

$$
\left\{\begin{array}{l}
q_{d}(t) \text { twice-differentiable } \\
q_{d}(t) \geq 0 \quad \text { for } t \in \Omega_{k}, k \geq 0 \\
q_{d}(t)=q_{d}\left(T_{1}^{2 k}\right) \exp \left[-\frac{c}{m}\left(t-T_{1}^{2 k}\right)\right] \\
\quad \text { for } t \in\left[T_{1}^{2 k}, t_{0}^{k}\right], k \geq 0 \\
q_{d}(t) \equiv 0 \text { on } \Omega_{2 k+1} .
\end{array}\right.
$$

The signal $q_{d}(t)$ is therefore defined such that when a constrained phase is desired, the free-motion phase ends with an exponentially decreasing $q_{d}(t)$. It is assumed that the time $T_{1}^{2 k}<t_{0}^{k}$ is such that no collision has occurred for $t<T_{1}^{2 k}$, 


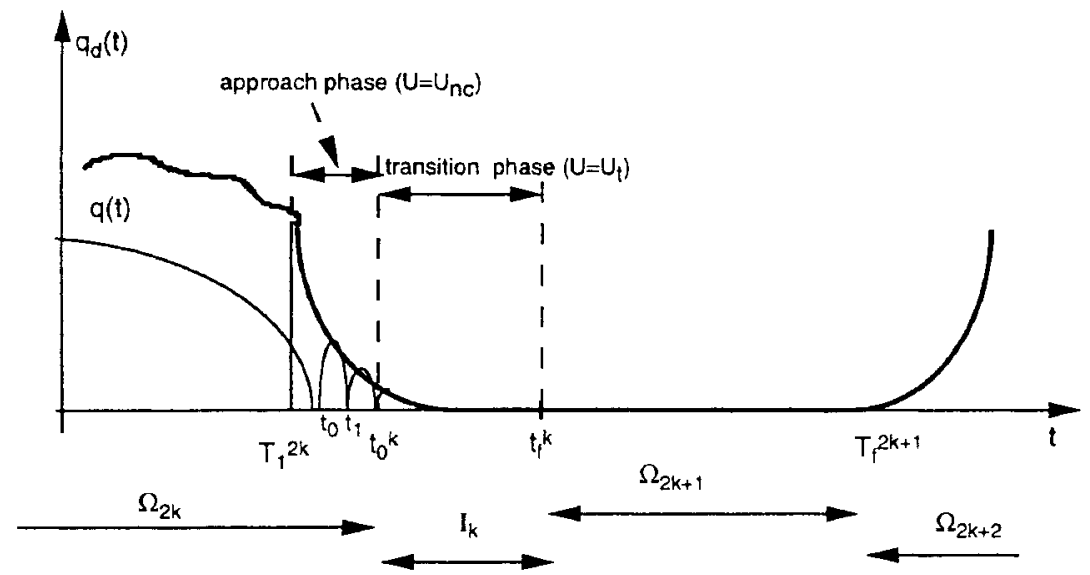

Fig. 1. Desired trajectory and switching times (weak stability).

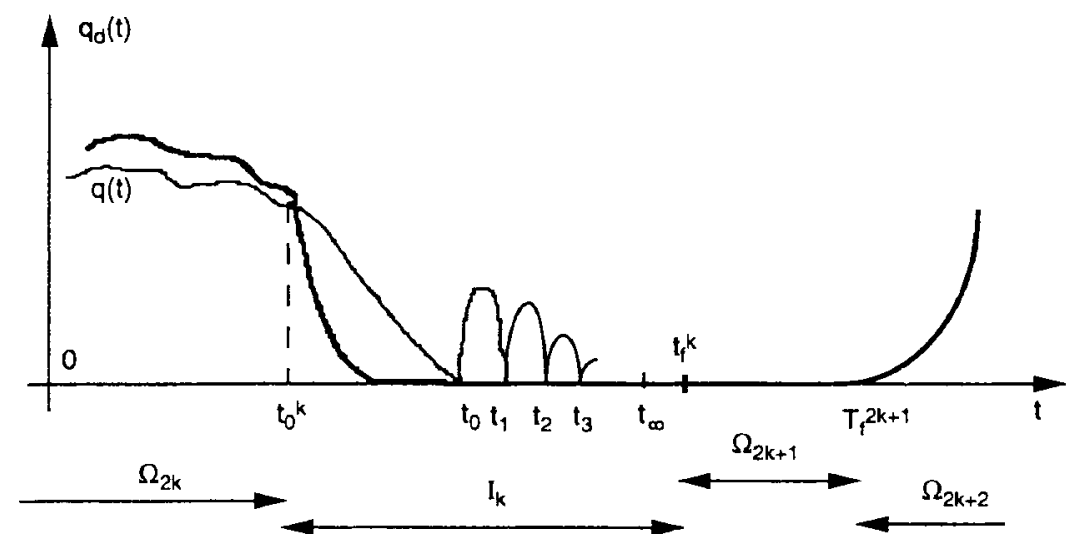

Fig. 2. Desired trajectory and switching times (strong stability).

$t \in \Omega_{2 k}$. The switching time $t_{0}^{k}$ has to be chosen $\geq T_{1}^{2 k}$. The rationale behind the form of $q_{d}(t)$ on $\left[T_{1}^{2 k}, t_{0}^{k}\right]$ will appear clearly from the calculation of $\sigma_{V}\left(t_{k}\right)$ [see (38)] during the free-motion phases $\Omega_{2 k}$, which has to be negative from the conditions in Lemma 1. Let us notice that given this choice of the approach period in $\Omega_{2 k}$, it is necessary to apply $U_{t}$ in (31) to obtain finite-time stabilization on $q=0$. Furthermore, the transition phase $I_{k}$ is chosen to end at a finite time $t_{f}^{k}$ such that the sequence of rebounds of the mass has stopped and $q_{d}\left(t_{f}^{k}\right)=\dot{q}_{d}\left(t_{f}^{k}\right)=0$. Notice that the value of $t_{f}^{k}$ does not need to be known explicitly for the stability analysis. Only its existence is needed, since both controllers on $I_{k}$ and $\Omega_{2 k+1}$ are the same. Finally, it is noteworthy that $q_{d}(t)$ is not used in the control input on $I_{k}$ and $\Omega_{2 k+1}$. It is therefore to be considered as a virtual signal on these intervals, used only for stability purposes. This means in particular that one does not need to calculate explicitly $q_{d}(t)$ on $\left[t_{0}^{k}, T_{f}^{2 k+1}\right]$. Only its existence as a twice-differentiable signal is needed. This remark is also true for the signal $q_{d}(t)$ and the switching times used in the next section; see Fig. 2. Further insight on the choice of $q_{d}(t)$ is given in Remark 7.

We shall also consider the positive definite function

$$
\begin{aligned}
V(x) & =V(\tilde{q}, \dot{\tilde{q}}) \\
& =\frac{1}{2} m \dot{\tilde{q}}^{2}+\frac{1}{2} \lambda_{2} \tilde{q}^{2}+c \tilde{q} \dot{\tilde{q}} \\
& =x^{T} P x
\end{aligned}
$$

with $0<c<\sqrt{m \lambda_{2}}$ and $x^{T}=(\tilde{q}, \dot{\tilde{q}})$. Hence the closed-loop equation on $\Omega_{2 k}, k \geq 0$ is given by

$$
\left\{\begin{aligned}
m \ddot{\tilde{q}}+\lambda_{1} \dot{\tilde{q}}+\lambda_{2} \tilde{q} & =0 \\
\sigma_{\dot{\tilde{q}}}\left(t_{k}\right) & =-(1+e) \dot{\tilde{q}}\left(t_{k}^{-}\right)-(1+e) \dot{q}_{d}\left(t_{k}\right) \\
& =\sigma_{\dot{q}}\left(t_{k}\right)
\end{aligned}\right.
$$

which represents a measure differential equation with fixed point $\tilde{q}=\dot{\tilde{q}}=0$, and impulsive disturbances $m \sigma_{\tilde{\tilde{q}}}\left(t_{k}\right) \delta_{t_{k}}=$ $P_{q}\left(t_{k}\right) \delta_{t_{k}}$, i.e., (35) can be rewritten as

$$
m \ddot{\tilde{q}}+\lambda_{1} \dot{\tilde{q}}+\lambda_{2} \tilde{q}=m \sum_{k} \sigma_{\tilde{\tilde{q}}}\left(t_{k}\right) \delta_{t_{k}}
$$

As we discussed in Section III, if $q_{d}(t)<0$, then it makes no sense to speak of Lyapunov stability for (35) or (36) because the fixed point (if any) belongs to $\partial \Phi \times \mathbb{R}$, i.e., here $q=\dot{q}=$ 0 . When $q_{d}(t)>0$, this may be done following the tools developed in [1].

Notice that due to the switching function $\alpha(t)$ in (32), the transition phase dynamics are

$$
m \ddot{q}=-F_{d}, \quad q \geq 0
$$

together with the restitution rule in (8). Hence the sequence $\left\{t_{k}\right\}$ of impact times is guaranteed to exist with a finite 
accumulation point $t_{\infty}$ (exactly the dynamics of the bouncing ball; see, e.g., [35]). Let us analyze the variations of $V$ in (34).

1) On $\Omega_{2 k}$ [see (35)]: $\dot{V} \leq-\gamma(\|x\|)$ for suitable choice of $\lambda_{1}>0, \lambda_{2}>0$. Moreover, on $\left[T_{1}^{2 k}, T_{f}^{2 k}\right]=\left[T_{1}^{2 k}, t_{0}^{k}\right]$ one obtains

$$
\begin{aligned}
\sigma_{V}\left(t_{k}\right)= & \frac{1}{2} m\left(e^{2}-1\right)\left[\dot{q}\left(t_{k}^{-}\right)\right]^{2}+(1+e) \dot{q}\left(t_{k}^{-}\right) \\
& \cdot\left[m \dot{q}_{d}\left(t_{k}\right)+c q_{d}\left(t_{k}\right)\right] \\
= & \frac{1}{2} m\left(e^{2}-1\right)\left[\dot{q}\left(t_{k}^{-}\right)\right]^{2} \\
\leq & 0
\end{aligned}
$$

for all $e \in[0,1)$ and $q_{d}(t)$ as in (33).

2) On $\Omega_{2 k+1}: V \equiv \dot{V} \equiv 0$, since $\widetilde{q} \equiv \dot{\tilde{q}} \equiv 0$ (see Fig. 1).

3) On $I_{k}$ [see (37)]: $V\left(t_{f}^{k}\right)=0 \leq V\left(t_{0}^{k}\right)=\frac{1}{2} m\left[\dot{\tilde{q}}\left(t_{0}^{k}\right)\right]^{2}+$ $\frac{1}{2} \lambda_{2}\left[q\left(t_{0}^{k}\right)\right]^{2}+c q\left(t_{0}^{k}\right) \dot{\tilde{q}}\left(t_{0}^{k}\right) \geq 0$, and $V$ is uniformly bounded on $I_{k}, k \geq 0$.

Remark 5: The usefulness of the exponentially decaying $q_{d}(t)$ in (33) on the interval $\left[T_{1}^{2 k}, t_{0}^{k}\right]$ clearly appears in (38). This approach phase guarantees that the MDE in (36) satisfies the requirements of Lemma 1 . This can be useful to define grazing trajectories $q_{d}(t)$ satisfying $m \dot{q}_{d}(t)+c q_{d}(t)=\gamma>$ 0 . Eventual shocks with the surface $q=0$ do not destroy the Lyapunov stability of the scheme for such $q_{d}(t)$.

Thus the following is true.

Claim 1: The system in (28) in closed loop with the controller in (29)-(32) and the switching times and $q_{d}(t)$ as in Fig. 1, is weakly stable.

Notice that a stronger result is obtained, i.e.: $\tilde{q}(t) \equiv \dot{\widetilde{q}}(t) \equiv 0$ for $t \in \Omega_{k}, k \geq 1$. This is due to the fact that we assume that the constraint position is known $(q=) 0$. When the second free-motion phase $\Omega_{2}$ starts, the initial conditions satisfy $\tilde{q}\left(T_{0}^{2}\right)=\dot{\tilde{q}}\left(T_{0}^{2}\right)=0$. Hence, there is a perfect subsequent tracking. This is obviously an ideal situation which will not occur in practice. The assumption that the constraints $F(q)=$ 0 are known is nevertheless a current assumption in basic theoretical studies [5], [7] of force/position control. As noted in Remark 3, the input $U_{t}$ could be defined such that on $I_{k}$, $t_{k+1}=t_{k}+T, k \leq N<+\infty$, before an eventual stabilization on the constraint or before another free-motion phase. In the latter case one has to define $t_{f}^{k}$ such that $V\left(t_{f}^{k}\right) \leq V\left(t_{0}^{k}\right)$, but $V\left(t_{f}^{k}\right)$ may be strictly positive since the system keeps bouncing on the surface during $I_{k}$.

Remark 6: Assume that $U=U_{n c}$ for all $t \geq 0$, with $q_{d}<$ 0 a constant signal. Strictly speaking, there is no free-motion phase since the fixed point of the free-motion closed-loop equation does not belong to $\operatorname{Int}(\Phi)=(0,+\infty) \times \mathbb{R}$. Hence this task (PD control and a constraint) reduces to $\mathbb{R}^{+}=$ $I_{0} \cup \Omega_{0}$, where $\lambda\left[I_{0}\right]<+\infty$ and $\lambda\left[\Omega_{0}\right]=+\infty$ if the transition phase is stable. $\Omega_{0}$ is the constrained phase. First note that since the unconstrained system is globally asymptotically stable with the Lyapunov function $V$ in (34), the sequence $\left\{t_{k}\right\}$ exists, because each time the system verifies $q(t)>0$ it tends to reach the constraint. After each rebound this is true. From the fact that the dynamics are similar to those of a bouncing ball with dissipation during the flight times, one supposes that $t_{\infty}<+\infty$ so that one can choose $I_{k}$ with $t_{f}^{0}<+\infty$. Then the positive definite function $V_{0}$ obtained by taking $q_{d} \equiv 0$ in (34) satisfies the requirements of Lemma 1. One therefore sees that the conditions of Lemma 1 do not necessarily require that a switching (or discontinuous) controller be applied. This depends on the task.

\section{B. Strongly Stable Task}

In this section, we examine how the transition phase controller $U_{t}$ and the desired trajectory influence the stability of the discrete mapping $P_{\Sigma}$ during the transition phase. From (38) it follows that if $q_{d}(t) \equiv 0$ then condition i) of Lemma 2 is satisfied. Condition ii) can be calculated as follows:

$$
\begin{aligned}
\int_{\left(t_{k}, t_{k+1}\right)} \dot{V}(t) d t \\
=V\left(t_{k+1}^{-}\right)-V\left(t_{k}^{+}\right) \\
=\int_{\left(t_{k}, t_{k+1}\right)}\left\{\dot{\tilde{q}}\left[U-m \ddot{q}_{d}\right]+\lambda_{2} \tilde{q} \dot{\tilde{q}}\right. \\
\left.\quad+c \dot{\tilde{q}}^{2}+c \tilde{q}\left[\frac{U}{m}-m \ddot{q}_{d}\right]\right\} d t \\
=\int_{\left(t_{k}, t_{k+1}\right)}\left\{\left(\dot{\tilde{q}}+\frac{c}{m} \tilde{q}\right)\left[U-m \ddot{q}_{d}\right]+c \dot{\tilde{q}}^{2}\right\} d t \\
\quad+\frac{\lambda_{2}}{2}\left[q_{d}^{2}\left(t_{k+1}\right)-q_{d}^{2}\left(t_{k}\right)\right] .
\end{aligned}
$$

If $q_{d} \equiv 0$ on $\left(t_{k}, t_{k+1}\right)$, then we get

$$
V\left(t_{k+1}^{-}\right)-V\left(t_{k}^{+}\right)=\int_{\left(t_{k}, t_{k+1}\right)}\left\{\left(\dot{q}+\frac{c}{m} q\right) U+c \dot{q}^{2}\right\} d t .
$$

One has to find out $U(t)$ such that the sequence $\left\{t_{k}\right\}$ exists with finite accumulation point and such that the right-hand side in (40) is negative. Let us consider ${ }^{11}$

$$
U_{t}(\dot{q})=-(\lambda+c) \dot{q}-F_{d}, \quad F_{d}>0, \lambda>0 .
$$

Notice first that introducing (41) into (28), we obtain the transition phase closed-loop equation in (20)

$$
m \ddot{q}+(\lambda+c) \dot{q}=-F_{d} .
$$

Similarly, as in (37) one sees from (42) that the fixed point of the closed-loop equation on $I_{k}$ belongs to $\partial \Phi \times \mathbb{R}=\{0\} \times \mathbb{R}$. Hence

$$
\begin{aligned}
q(t)= & {\left[\frac{F_{d}}{\lambda+c}+\dot{q}\left(t_{0}^{k}\right)\right] } \\
& \cdot \frac{m}{\lambda+c}\left\{\exp \left[-\frac{\lambda+c}{m}\left(t-t_{0}^{k}\right)\right]-1\right\} \\
& +\frac{F_{d}}{\lambda+c}\left(t-t_{0}^{k}\right)+q\left(t_{0}^{k}\right) .
\end{aligned}
$$

It follows that $q(t) \rightarrow-\infty$ as $t \rightarrow+\infty$, hence there exists a shock instant $t_{k}$ such that $\dot{q}$ is discontinuous at $t_{k}$. Then after $t_{k}, q(t)=\left[F_{d} /(\lambda+c)+\dot{q}\left(t_{k}^{+}\right)\right][m /(\lambda+c)](\exp \{-[(\lambda+$ $\left.\left.c) / m]\left(t-t_{k}\right)\right\}-1\right)+\left[F_{d} /(\lambda+c)\right]\left(t-t_{k}\right)$ so that another shock must occur at $t_{k+1}>t_{k}$. We deduce that $U_{t}$ in (41)

\footnotetext{
${ }^{11}$ It has been experimentally shown in [20] that velocity feedback improves the system's behavior during the transition phase. Hence the interest of considering $U_{t}$ in (41) instead of $U_{t}$ in (31).
} 
guarantees that the system collides the constraint after any rebound. Following the terminology in [28], the closed-loop system is a (nonautonomous) flow with collisions, i.e., the concatenation of a flow (dynamics during flight-times) and a diffeomorphism [restitution law at collisions; see (4)], which are both dissipative. The dissipativity of the diffeomorphism can guarantee some finite-time convergence properties due to the impulsive behavior at collisions. It is noteworthy that the Poincaré map associated with (42) and the section $q=0$ cannot be calculated explicitly. Indeed, this would require the calculation of the impact times $t_{k}$, which are given by $q\left(t_{k}\right)=$ 0 , i.e.,

$$
\begin{aligned}
& {\left[\frac{F_{d}}{\lambda+c}+\dot{q}\left(t_{k}^{+}\right)\right] \frac{m}{\lambda+c}\left\{1-\exp \left[-\frac{\lambda+c}{m}\left(t_{k+1}-t_{k}\right)\right]\right\}} \\
& -\frac{F_{d}}{\lambda+c}\left(t_{k+1}-t_{k}\right)=0 .
\end{aligned}
$$

Equation (44) is an implicit equation for the flight-time $\Delta_{k+1} \triangleq t_{k+1}-t_{k}$. In the case of a bouncing ball, the dissipation is generally assumed to be zero $(\lambda+c=0)$, and the flight-times are easily calculable as $\Delta_{k+1}=\left(2 \mathrm{~m} / F_{d}\right) \dot{q}\left(t_{k}^{+}\right)$ (that can be obtained from (44) by taking the limit as $\lambda+c \rightarrow 0$ and eliminating the solution $\Delta_{k+1}=0$ ), which allows us to obtain the sequences $\left\{\Delta_{k}\right\}$ and $\left\{\dot{q}\left(t_{k}^{+}\right)\right\}$explicitly. Now let us conjecture the continuity of the solutions of (8) and (42) with respect to the parameters, in particular $\lambda+c$. Then the sequence $\left\{t_{k}\right\}$ must have a finite accumulation point for all $e \in[0,1) .{ }^{12}$ Moreover, the impact of the Poincare map $P_{\Sigma}: \dot{q}\left(t_{k}^{+}\right) \mapsto \dot{q}\left(t_{k+1}^{+}\right)$fixed point stability is provable as shown next, although $P_{\Sigma}$ is not calculable explicitly.

Indeed, one gets from (40) and (41)

$$
\begin{aligned}
V\left(t_{k+1}^{-}\right)-V\left(t_{k}^{+}\right)= & -\int_{\left(t_{k}, t_{k+1}\right)} \lambda \dot{q}^{2}(t) d t-F_{d}[q(t)]_{t_{k}}^{t_{k+1}} \\
& -c \frac{\lambda+c}{2 m}\left[q^{2}(t)\right]_{t_{k}}^{t_{k+1}} \\
& -\frac{c}{m} F_{d} \int_{t_{k}}^{t_{k+1}} q(t) d t \\
\leq & -\lambda \int_{\left(t_{k}, t_{k+1}\right)} \dot{q}^{2}(t) d t \\
\leq & 0
\end{aligned}
$$

since $q(t)>0$ on $\left(t_{k}, t_{k+1}\right)$ and $F_{d}>0, c>0, q\left(t_{k}\right)=0$. Hence condition ii) in Lemma 2 is verified if $U=U_{t}$ as in (41). Now since $q_{d} \equiv 0$, it follows from (38) that i) in Lemma 2 is verified also. Moreover, notice that $V_{\Sigma}(\dot{q})=\frac{1}{2} m \dot{q}^{2}$ so that $V_{\Sigma}(0)=0$. We conclude that the Poincaré map $P_{\Sigma}$ associated with the system in (28) in closed-loop form with (41) has a Lyapunov stable fixed point $\dot{q}^{\star}=0$ with Lyapunov

\footnotetext{
${ }^{12}$ This is the classical bouncing ball case with dissipation during flighttimes. We conjecture in fact that $t_{\infty}$ is a continuous function of $\lambda+c$. Since $t_{\infty}(0)<+\infty$, then $t_{\infty}(\lambda+c)$ must be bounded for $\lambda+c>0$ also. Although there is no rigorous proof of this to date, it would be quite surprising that the addition of any small amount of dissipativity during flight times destroys the finite time convergence property. Anyway, we can always guarantee the finite time stabilization by switching to $U_{t}$ in (31) after a time $t_{0}^{k}+\delta$. But then strong stability is lost.
}

function $V_{\Sigma}(\dot{q})$. We have thus transformed the continuous-time Lyapunov function (for free-motion phases) $V$ in (34) into a reduced-order discrete-time Lyapunov function (for impact or transition phases) $V_{\Sigma}$. However, it remains to determine how to choose $q_{d}(t)$ to get $q_{d} \equiv 0$ on $I_{k}$. A suitable signal $q_{d}(t)$ and switching times are depicted in Fig. 2. Let us recall that on $I_{k}$ and $\Omega_{2 k+1}$, only the existence of $q_{d}(t)$ as a twice differentiable signal is needed.

The main discrepancy between $q_{d}(t)$ and the switching times in Figs. 1 and 2 is that in the second case, the approach phase is entirely included into $I_{k}$. The controller $U_{t}$ is therefore switched on earlier. In the first case, the approach phase was partially included in $\Omega_{2 k}$, which implies a particular form of $q_{d}(t)$ to comply with Lemma 1 requirements.

We thus have proved the following.

Claim 2: The system in (28) in closed-loop form with the controller in (29), (30), (32), (41), the switching times, and $q_{d}(t)$ as in Fig. 2, is strongly stable.

Remark 7-About the Choice of the Desired Trajectory $q_{d}(t)$ : It follows from the conditions of Lemma 1 that one must have $q_{d}(t) \equiv 0$ on $\Omega_{2 k+1}$ (constrained phases), and from Lemma 2 this must hold also on $I_{k} \cdot{ }^{13}$ On one hand, it is needed to satisfy $V\left(t_{f}^{k}\right) \leq V\left(t_{0}^{k}\right)$. If $q_{d}\left(t_{f}^{k}\right) \neq 0$, then $V\left(t_{f}^{k}\right)=\frac{1}{2} m\left[\dot{q}_{d}\left(t_{f}^{k}\right)\right]^{2}+\frac{1}{2} \lambda_{2}\left[q_{d}\left(t_{f}^{k}\right)\right]^{2}+c \dot{q}_{d}\left(t_{f}^{k}\right) q_{d}\left(t_{f}^{k}\right)>0$. Now for all $\varepsilon>0$, for all $\eta>0$, there exists $T \in \Omega_{2 k}$. such that for all $t \geq T, t \in \Omega_{2 k}$, and for all $\left\|x\left(T_{0}^{2 k}\right)\right\| \leq \eta$, one has $V(t)<\varepsilon$. In other words the system may remain long enough in the free-motion phase $\Omega_{2 k}$ so that the required inequality in Lemma 1 may never be satisfied. On the other hand, application of Lyapunov techniques to study the stability of the Poincaré map fixed point $\bar{x}_{\Sigma}^{\star}$ requires $V_{\Sigma}\left(\bar{x}_{\Sigma}^{\star}\right)=0$ [39]. This result may appear as a consequence of the choice of the positive definite function $V$ in (34). Notice, nevertheless, that to guarantee negative definiteness of $\dot{V}$ on $\Omega_{2 k}$, this choice is the only possible one. Moreover, the fact that the desired trajectory is consistent with the constraints is a logical feature.

Finally, we could have defined $q_{d}(t)$ that smoothly converges to zero in finite time, with $q_{d}(t) \geq 0$ for all $t \geq 0$, applying $U_{n c}$ as long as $q_{d}>0$ (for instance as in Fig. 2, but choosing $t_{0}^{k}$ such that $q_{d}\left(t_{0}^{k}\right)=0$ ). From a practical point of view, this may not be a good choice since it means that the robot has to slow down and attain the constraint with a zero velocity on $\Omega_{2 k}, k \geq 1$. Such a strategy is quite timeconsuming. For the sake of the generality of the analysis, we consider in this paper less smooth strategies which involve shocks between the robot's tip (a simple mass for the moment) and the obstacle. Note that if the constraint position is not well known, then impacts are likely to occur. It is therefore much more interesting to consider collisions in a first theoretical framework. Moreover, impacts may be desired in certain robotic tasks. Our goal in this study is therefore not to design a controller such that no collisions occur in the system. If this can be done, the stability framework can still be used. It is

\footnotetext{
${ }^{13}$ But notice that the conditions of Lemma 1 do not imply that $q_{d}(t)=0$ on $I_{k}$.
} 
clear that a necessary and sufficient condition for no impact to occur is that $\dot{q}_{\text {norm }}\left(t_{k}^{-}\right)=0$ [see (11)].

\section{Dynamic State Feedback}

It is interesting to notice that the proposed stability concept allows for dynamic state feedback controllers to be considered. Let us consider the control input

$$
\begin{aligned}
U\left(t, q, \dot{q}, z_{1}, z_{2}\right)= & \alpha_{1}(t) U_{n c}\left(t, q, \dot{q}, z_{1}\right)+\alpha_{2}(t) U_{t}(\dot{q}) \\
& +\alpha_{3}(t) U_{c}\left(z_{2}\right)
\end{aligned}
$$

where $q_{d}(t)$ is depicted in Figs. 1 or 2, and

$$
\begin{aligned}
U_{n c}\left(t, q, \dot{q}, z_{1}\right) & =m \ddot{q}_{d}-\lambda_{1} \dot{\tilde{q}}-\lambda_{2} \tilde{q}-\lambda_{3} z_{1} \\
\dot{z}_{1} & = \begin{cases}\tilde{q}(t) & \text { on } \Omega_{2 k} \\
h(t) & \text { on } I_{k} \\
0 & \text { on } \Omega_{2 k+1}\end{cases}
\end{aligned}
$$

with $h(t)$ such that $z_{1}\left(t_{0}^{k}+\delta\right)=0$ for some $\delta>0, t_{0}^{k}+\delta<t_{0}$, and such that $z_{1}(t)$ is smooth enough

$$
U_{t}(\dot{q})=-\lambda_{4} \dot{q}-F_{d}
$$

$\lambda_{4}>0$

$$
\begin{aligned}
U_{c}\left(z_{2}\right) & =-F_{d}+z_{2} \\
\dot{z}_{2} & = \begin{cases}F-F_{d} & \text { on } \Omega_{2 k+1} \\
0 & \text { on } I_{k} \\
0 & \text { on } \Omega_{2 k} .\end{cases}
\end{aligned}
$$

$F$ is the force exerted by the constraint on the mass, and

$$
\begin{aligned}
& \alpha_{1}(t)= \begin{cases}1 & \text { if } t \in \Omega_{2 k} \\
0 & \text { otherwise }\end{cases} \\
& \alpha_{2}(t)= \begin{cases}1 & \text { if } t \in I_{k} \\
0 & \text { otherwise }\end{cases} \\
& \alpha_{3}(t)= \begin{cases}1 & \text { if } t \in \Omega_{2 k+1} \\
0 & \text { otherwise. }\end{cases}
\end{aligned}
$$

Let us consider the positive-definite function

$$
\begin{aligned}
V(x) & =V\left(z, z_{2}\right) \\
& =z^{T} P z+\frac{1}{2} z_{2}^{2} \\
& =x^{T}\left(\begin{array}{ll}
P & 0 \\
0 & 1
\end{array}\right) x
\end{aligned}
$$

with $z^{T}=\left(z_{1}, \tilde{q}, \dot{\tilde{q}}\right), x^{T}=\left(z^{T}, z_{2}\right), P>0$ is the solution of $A^{T} P+P A=-Q, Q>0$, where $\dot{x}=A x$ is the statespace representation of $m \ddot{q}=U_{n c}\left(t, q, \dot{q}, z_{1}\right), A$ is a Hurwitz matrix for a suitable choice of $\lambda_{1}, \lambda_{2}, \lambda_{3}>0$. It can be shown that the conditions of Lemma 1b) are satisfied with $\xi_{1}=z$ and $\xi_{2}=z_{2}, \Omega^{1}=\cup_{k} \Omega_{2 k}$, and $\Omega^{2}=\cup_{k} \Omega_{2 k+1}$. Depending on the chosen $q_{d}(t)$ and switching times as depicted in Figs. 1 and 2, and a suitable $\lambda_{4}>0$, the closed-loop scheme may be weakly or strongly stable; see [64].
Remark 8: The controllers in (29)-(32), Fig. 1, and (29), (30), (32), (41), and Fig. 2 are discontinuous (measurable) in $t$ and Lipschitz continuous in the state variables. They verify Carathéodory-like conditions. Hence the existence of the closed-loop solution is guaranteed for all $t \geq 0$ from [9, Th. 2] with the modifications we discussed in Section II. ${ }^{14}$ The dynamic feedback in (46) yields a closed loop that does not exactly fit within that framework. However, since it involves only integrators, and thus additional continuous signals, the extension should be possible.

\section{V. n-DOF RIGID MANIPULATORS}

In this section, we discuss the extension of the one-dof case to $n$-dof manipulators. In particular we highlight the consequences of the generalized restitution rule defined in Section II-A-2, through the generalized velocity transformation in (11). It is shown that although weak stability can in general be obtained, strong stability is more difficult to achieve when the controller is designed from generalized coordinates whose derivative does not correspond to the transformed velocity in (11).

\section{A. Integrable Transformed Velocities}

Consider the rigid $n$-dof manipulator dynamics in (1), the generalized velocities transformation in (11), and in (2) orthogonal constraints $F_{1}(q) \geq 0, F_{2}(q) \geq 0$. Then one can write the dynamical equation as

$$
\begin{array}{r}
\ddot{q}_{\text {norm }}-E_{1} \dot{\mathcal{M}} \dot{q}+n_{q}^{T}[C(q, \dot{q}) \dot{q}+g(q)]=n_{q}^{T} U \\
\ddot{q}_{\text {tang }}-E_{2} \dot{\mathcal{M}} \dot{q}+t_{q}^{T}[C(q, \dot{q}) \dot{q}+g(q)]=t_{q}^{T} U
\end{array}
$$

Equation (13) is true for $t=t_{k}$, and

$$
\mathcal{M}=\left[\begin{array}{l}
n_{q}^{T} \\
t_{q}^{T}
\end{array}\right] M(q) .
$$

The restitution rules are defined in (14),

$$
\begin{aligned}
& E_{1}=\left[I_{2} \vdots 0_{2 \times(n-2)}\right] \\
& E_{2}=\left[0_{(n-2) \times 2} \vdots I_{n-2}\right] .
\end{aligned}
$$

It clearly appears from (54) and (13) why the orthogonality of the constraints allows us to treat the $n$-dof case as $n$ onedof cases. Between shocks there is a coupling between the $n$ equations in (54) through the Coriolis and gravity terms, but it can be compensated for via suitable feedback.

Let us apply a linearizing and decoupling control input $U$ such that

$$
\begin{aligned}
{\left[\begin{array}{c}
n_{q}^{T} \\
t_{q}^{T}
\end{array}\right] U=} & {\left[\begin{array}{c}
n_{q}^{T} \\
t_{q}^{T}
\end{array}\right][C(q, \dot{q}) \dot{q}+g(q)] } \\
& -\left[\begin{array}{c}
E_{1} \dot{\mathcal{M}} \dot{q} \\
E_{2} \dot{\mathcal{M}} \dot{q}
\end{array}\right]+v
\end{aligned}
$$

\footnotetext{
${ }^{14}$ Notice, nevertheless, that $e \in(0,1]$ in $[9$, Th. 2], i.e., plastic shocks are not treated.
} 
Hence we obtain

$$
\left\{\begin{array}{l}
\ddot{q}_{\text {norm }, 1}=v_{1} \\
\ddot{q}_{\text {norm }, 2}=v_{2} \\
\ddot{q}_{\text {tang }, 1}=v_{3} \\
\vdots \\
\ddot{q}_{\text {tang, } \mathbf{n}-2}=v_{n} .
\end{array}\right.
$$

Let us assume that the system under consideration is written in generalized coordinates as in Remark 4, i.e., the unilateral constraints are simply $q_{1} \geq 0, q_{2} \geq 0$, and the generalized velocity transformation in (11) is defined using a basis $\left(n_{q}, t_{q}\right)$ without normalization of $n_{q, i}$ in (10). Indeed the definition of normalized vectors $n_{q, i}$ in (10) is convenient for instance to write down the kinetic energy loss at impacts in a quite simple form; see Section II. But it is not necessarily suitable for control purposes, i.e., for $\mathcal{M}$ to be a Jacobian. Most importantly, let us do the assumption that there exist functions $q_{t}(t)$ such that $\dot{q}_{t}=\dot{q}_{\text {tang }}$. Notice that $\dot{q}_{\text {norm, } \mathrm{i}}=\varepsilon_{i}^{T} \dot{q}=\dot{q}_{i}$ so that $q_{\text {norm, } \mathrm{i}}=q_{i}, \Sigma_{i}=\left\{x: q_{\text {norm, } \mathrm{i}}=0\right\}, i=1,2$. More generally, one would obtain $q_{\text {norm, } \mathrm{i}}=F_{i}(q)$. Now let us define

$$
\begin{aligned}
v_{i}= & \alpha_{1}(t) U_{n c}\left(t, q_{i}, \dot{q}_{i}, z_{1, i}\right)+\alpha_{2}(t) U_{t}\left(\dot{q}_{i}\right) \\
& +\alpha_{3}(t) U_{c}\left(z_{2, i}\right)
\end{aligned}
$$

for $i=1,2$, where all the terms are defined similarly as in (47) through (52), and

$$
v_{i}=\ddot{q}_{t, i}^{d}-\gamma_{2} \dot{\tilde{q}}_{t, i}-\gamma_{1} \tilde{q}_{t, i}
$$

for $i=3, \cdots, n$, where $\gamma_{1}, \gamma_{2}$ are suitably chosen such that the polynomial $m s^{2}+\gamma_{1} s+\gamma_{2}$ is Hurwitz. Let us choose

$$
\begin{gathered}
V(x)=z_{1}^{T} P_{1} z_{1}+z_{2}^{T} P_{2} z_{2}+\frac{1}{2} z_{2,1}^{2} \\
+\frac{1}{2} z_{2,2}^{2}+\sum_{k=3}^{n} z_{k}^{T} P_{k} z_{k}
\end{gathered}
$$

where $x^{T}=\left(z_{1}^{T}, z_{2}^{T}, z_{2,1}, z_{2,2}, z_{3}^{T}, \cdots, z_{n}^{T}\right)$. The function $V$ in (59) can be written as $V=V_{1}\left(z_{1}, z_{2,1}\right)+V_{2}\left(z_{2}, z_{2,2}\right)+$ $\sum_{k=3}^{n} V_{k}\left(z_{k}\right)$, where $z_{k}^{T}=\left(\tilde{q}_{t, k}, \dot{\tilde{q}}_{t, k}\right), z_{i}^{T}=\left(z_{1}, i, \tilde{q}_{i}, \check{\tilde{q}}_{i}\right)$, $i=1,2$. The $P_{i}$ 's are naturally defined from the Lyapunov equations associated to each sub-closed-loop equation obtained by introducing (57) and (58) into (56). Suppose that the gains $\gamma_{1}$ and $\gamma_{2}$ in (58) are suitably chosen so that $\left\|z_{k}\right\|$ is always decreasing, $3 \leq k \leq n$. It is then not difficult to show that the result of Lemma 1b) applies, with $\xi_{1}^{T}=$ $\left(z_{1}^{T}, z_{2,1}, z_{3}, \cdots, z_{n}\right), \xi_{2}^{T}=\left(z_{2}^{T}, z_{2,2}\right)$, and $\Omega^{1}=\cup_{k} \Omega_{2 k}$, and $\Omega^{2}=\cup_{k} \Omega_{2 k+1}$. Indeed the last term $\sum_{k=3}^{n} V_{k}\left(z_{k}\right)$ in (59) evolves independently of the rest of $V$, and its derivative is given by $-z_{k}^{T} Q_{k} z_{k}, Q_{k}>0$, for all $t \geq 0$. Also the terms $V_{1}\left(z_{1}, z_{2,1}\right)$ and $V_{2}\left(z_{2}, z_{2,2}\right)$ evolve independently, one from each other. The controller guarantees that the system stabilizes in finite time on each surface $\Sigma_{1}, \Sigma_{2}$.

Strong stability is assured if the constraint is of codimension one. For the codimension-two case, let us note that (56) in closed-loop with (57), (58) satisfies the requirements of Lemma 2, provided both surfaces $\Sigma_{1}$ and $\Sigma_{2}$ are attained simultaneously during $I_{k}$. In other words, the system strikes the domain $\Phi$ exactly at the singularity of $\partial \Phi$. This may be guaranteed by taking the same initial conditions and switching times in both equations governing $q_{1}(t)$ and $q_{2}(t)$ evolution, and with restitution coefficients $e_{1}=e_{2}$. However, the surfaces $\Sigma_{1}$ and $\Sigma_{2}$ may in general be attained at different times, and in any order. If one guarantees that $V_{1}$ and $V_{2}$ decrease between any impacts, or if both functions satisfy $V_{i}\left(t_{k+1}^{-}\right) \leq V_{i}\left(t_{k}^{+}\right)$, then the system is strongly stable. This is difficult to obtain, however, because when the surface $F_{1}(q)$ is attained, $F_{2}(q)>0$ and vice-versa, which hampers to get an inequality like in (45). A simpler solution is to impose via a suitable choice of the desired trajectory that which the system first stabilizes on $\Sigma_{1}$, and then on $\Sigma_{2}$, which corresponds to two simple impacts. Since the dynamics are perfectly decoupled, such a choice is possible. However, the general problem of assuring strong stability with possible multiple impacts remains open, even in the case of integrable transformed velocities and orthogonal constraints.

\section{B. Nonintegrable Transformed Velocities}

What if $\mathcal{M}$ is not a Jacobian, which might be indeed the case in general? Then there exists no $q_{t}(t)$ such that $\dot{q}_{t}(t)=\dot{q}_{\text {tang }}$ in (11). It is still possible to have $\dot{q}_{\text {tang }}$ track some desired signal $\dot{q}_{\text {tang }}^{d}(t)$, which means that the components of $M(q) \dot{q}$ along $t_{q, i}, 1 \leq i \leq n-2$ (or in other words the components of $\dot{q}$ along $t_{q}, i$ and calculated with the kinetic metric) track $\dot{q}_{\text {tang }}^{d}(t)$. But the rest of the state vector (position) evolution is not clearly related to $q_{\text {tang }}^{d}(t)$ since $q(t)=q(0)+\int_{0}^{t} \mathcal{M}^{-1}[q(\tau)]\left[\begin{array}{l}\dot{q}_{\text {norm }} \\ \dot{q}_{\text {tang }}\end{array}\right] d \tau$. On the other hand it is difficult to design the overall scheme following the mixed discrete continuous-time stability analysis of Lemma 2, using for instance the coordinates introduced in [5] (see Remark 4). Indeed, during the rebounds phase, the velocity components $\dot{x}_{2}$ will in general possess jumps at $t_{k}$. Suppose that $x_{1} \in \mathbb{R}$. Following [5] we get in the $X$-coordinates the dynamical equations ${ }^{15}$

$$
\begin{aligned}
\bar{M}_{11} \ddot{x}_{1}+\bar{M}_{12} \ddot{x}_{2}+H_{1}(q, \dot{q}) & =T_{1} U+T_{1} P_{q} \\
& =T_{1} U+P_{X} \\
\bar{M}_{21} \ddot{x}_{1}+\bar{M}_{22} \ddot{x}_{2}+H_{2}(q, \dot{q}) & =T_{2} U
\end{aligned}
$$

where the various terms come from the nonsingular transformation between $X$ and $q$ and are defined in [5]. In particular, $\bar{M}_{i j}=E_{i} \bar{M} E_{j}^{T}$, for $i=1,2, j=1,2, \bar{M}$ is the inertia matrix in $X$-coordinates, and

$$
E_{1}=\left[1 \vdots 0_{1 \times(n-1)}\right], \quad E_{2}=\left[0_{(n-1) \times 1} \vdots I_{n-1}\right] .
$$

Thus one has $\bar{M}_{11}>0 \in \mathbb{R}, \bar{M}_{12}=\bar{M}_{21}^{T} \in \mathbb{R}^{1 \times(n-1)}$, and $\bar{M}_{22}>0 \in \mathbb{R}^{(n-1) \times(n-1)}$. The terms $H_{1}(q, \dot{q})$ and $H_{2}(q, \dot{q})$ contain the centrifugal and centripetal terms, $T_{1} \in \mathbb{R}^{1 \times n}$ and $T_{2} \in \mathbb{R}^{(n-1) \times n}$ are such that

$$
\left[\begin{array}{l}
T_{1} \\
T_{2}
\end{array}\right]
$$

is full rank. The vector $P_{X}$ in (60) generically represents the interaction force, i.e., $P_{X}=\nabla_{X} F(X) p_{x, 1}$, and $p_{x, 1} \in \mathbb{R}^{+}$

\footnotetext{
${ }^{15}$ For the sake of briefness of the paper and since the McClamroch-Wang transformation is now very well known, we do not provide details on it.
} 
is the Lagrange multiplier associated with $F(X)=0$. In a permanently constrained case $x_{1} \equiv 0$ and the second equation in (60) represents the motion along the constraint, see [5]. During a collision with the constraint, application of Newton's generalized restitution rule yields $\dot{x}_{1}\left(t_{k}^{+}\right)=-e \dot{x}_{1}\left(t_{k}^{-}\right)$since $\nabla_{X} F(X)^{T}=(1,0, \cdots, 0)$ (in the case of a codimension-one constraint). From (60) it therefore follows that

$$
\bar{M}_{21} \sigma_{\dot{x}_{1}}\left(t_{k}\right)+\bar{M}_{22} \sigma_{\dot{x}_{2}}\left(t_{k}\right)=0
$$

whereas

$$
\bar{M}_{11} \sigma_{\dot{x}_{1}}\left(t_{k}\right)+\bar{M}_{12} \sigma_{\dot{x}_{2}}\left(t_{k}\right)=p_{x, 1}\left(t_{k}\right) .
$$

Thus the jumps in $\dot{x}_{2}$ can be calculated from the jumps of $\dot{x}_{1}$ using the algebraic dynamics at impacts, as

$$
\begin{aligned}
\sigma_{\dot{x}_{2}}\left(t_{k}\right) & =-\bar{M}_{22}^{-1} \bar{M}_{21} \sigma_{\dot{x}_{1}}\left(t_{k}\right) \\
& =\bar{M}_{22}^{-1} \bar{M}_{21}(1+e) \dot{x}_{1}\left(t_{k}^{-}\right) .
\end{aligned}
$$

The percussion is then obtained from (62). The only case when $\sigma_{\dot{x}_{2}}\left(t_{k}\right)=0$ is when the inertia matrix in $X$-coordinates is block diagonal, i.e., $\bar{M}_{12}=0$. If such is the case, then the algebraic impact equation in (62) yields $\bar{M}_{11} \sigma_{\dot{x}_{1}}\left(t_{k}\right)=p_{x, 1}\left(t_{k}\right)$ and $\bar{M}_{22} \sigma_{\dot{x}_{2}}\left(t_{k}\right)=0$. Notice that the decoupling between $\ddot{x}_{1}$ and $\ddot{x}_{2}$ is independent of the orthogonality condition (4) between the constraints, which concerns here $\bar{M}_{11}$ when $x_{1} \in$ $\mathbb{R}^{m}, m \geq 2$. Indeed with $\bar{M}_{12}=\bar{M}_{21}=0$, the orthogonality condition implies $\varepsilon_{i}^{T} \bar{M}_{11}^{-1} \varepsilon_{j}=0, i, j \in \mathcal{I}, i \neq j$, i.e., $\bar{M}_{11}$ is diagonal.

We now illustrate the difficulty related to stabilization (in the sense of Lemmas 1 and 2) of a complete task for the system in (60). In what follows $\tilde{X}(t)=X(t)-X_{d}(t)$. The desired trajectories are $x_{2 d} \in \mathcal{C}^{2}\left[\mathbb{R}^{+}\right]$and $x_{1 d}$ can be chosen for instance as in Figs. 1 or 2. Let us choose the controllers as follows.

- On $\Omega_{2 k}$ : The control law is chosen as

$$
\left[\begin{array}{l}
T_{1} \\
T_{2}
\end{array}\right] U_{n c}=\bar{M}\left[\begin{array}{l}
v_{1} \\
v_{2}
\end{array}\right]+\left[\begin{array}{l}
H_{1} \\
H_{2}
\end{array}\right]
$$

with $v_{1} \in \mathbb{R}$ and $v_{2} \in \mathbb{R}^{n-1}$. The first closed-loop equation is simply $\ddot{x}_{1}=v_{1}$ and $\ddot{x}_{2}=v_{2}$

$$
\begin{aligned}
v & =\left[\begin{array}{l}
v_{1} \\
v_{2}
\end{array}\right] \\
& =\ddot{X}_{d}-\lambda_{1} \dot{\tilde{X}}-\lambda_{2} \tilde{X} .
\end{aligned}
$$

Hence the closed-loop equation is $\ddot{\tilde{X}}+\lambda_{1} \dot{\tilde{X}}+\lambda_{2} \tilde{X}=0$.

- On $\Omega_{2 k+1}$ : The open-loop system is obtained from (60) by taking $x_{1} \equiv 0$. The controller is chosen as

$$
\begin{aligned}
& T_{1} U_{c}=\bar{M}_{12} v_{2}+H_{1}-p_{x d} \\
& T_{2} U_{c}=\bar{M}_{22} v_{2}+H_{2}
\end{aligned}
$$

where $v_{2}=\ddot{x}_{2 d}-\lambda_{1} \dot{\tilde{x}}_{2}-\lambda_{2} \tilde{x}_{2}$. Hence the closed-loop is given by $x_{1} \equiv 0, \ddot{\widetilde{x}}_{2}+\lambda_{1} \dot{\widetilde{x}}_{2}+\lambda_{2} \tilde{x}_{2}=0$ and $p_{x, 1}=p_{x d}$.
The stability on $\Omega_{2 k}$ and $\Omega_{2 k+1}$ can be shown with the Lyapunov function $V=V_{1}\left(\tilde{x}_{1}, \dot{\widetilde{x}}_{1}\right)+V_{2}\left(\tilde{x}_{2}, \dot{\tilde{x}}_{2}\right) . V_{1}$ and $V_{2}$ are chosen similarly as in (34). Clearly on $\Omega_{2 k}$ and for a suitable choice of the gains $\lambda_{1}, \lambda_{2}, \dot{V} \leq-\gamma[\|(\tilde{X}, \dot{\tilde{X}})\|]$. On $\Omega_{2 k+1}$, if $x_{1 d} \equiv 0$, then $V_{1} \equiv 0$ and $\dot{\dot{V}}_{2} \leq-\gamma\left[\left\|\left(\tilde{x}_{2}, \tilde{\tilde{x}}_{2}\right)\right\|\right]$. Taking $\xi_{1}=x_{1}, \xi_{2}=x_{2}, \Omega^{1}=\cup_{k} \Omega_{2 k}, \Omega^{2}=\cup_{k} \Omega_{2 k+1}$, and choosing feedback gains $\lambda_{1}, \lambda_{2}$ such that $\left\|\left(x_{2}^{T}, \dot{x}_{2}^{T}\right)\right\|$ always decreases, the conditions required in b) of Lemma 1 are fulfilled.

- On $I_{k}$ : It remains now to design the controller on $I_{k}$ such that the condition $V\left(t_{f}^{k}\right) \leq V\left(t_{0}^{k}\right)$ is satisfied. Let us choose $U_{t}$ as in (64), but with $v_{1}=-a, a>$ 0 , and $v_{2}=\ddot{x}_{2 d}-\lambda_{1} \dot{\tilde{x}}_{2}-\lambda_{2} \tilde{x}_{2}$. The $x_{1}$-dynamics are therefore those of the vertical bouncing ball which guarantees that $x_{1}$ tends to zero in a finite time $t_{\infty}$. Indeed it can be calculated that the first shock occurs at $t_{0}=(1 / a) \dot{x}_{1}\left(t_{0}^{k}\right)+(1 / a) \sqrt{\dot{x}_{1}^{2}\left(t_{0}^{k}\right)+2 a x_{1}\left(t_{0}^{k}\right)}+t_{0}^{k}>t_{0}^{k}$. Then one obtains $\dot{x}_{1}\left(t_{k}^{+}\right)=-e^{k+1} \dot{x}_{1}\left(t_{0}^{-}\right)$and $\Delta_{k}=$ $t_{k}-t_{k-1}=(2 / a) e^{k} \dot{x}_{1}\left(t_{0}^{-}\right)$. From (63) one sees that the jumps in $\dot{x}_{2}$ also converge exponentially toward zero in finite time (provided of course $e<1$ ). They are given by $\sigma_{\dot{x}_{2}}\left(t_{k}\right)=a[(e+1) / 2] \bar{M}_{22}^{-1} \bar{M}_{21} \Delta_{k}$.

The function $V_{1}$ can be analyzed exactly as we did for $V$ in (34). But contrarily to the integrable velocity case, $V_{2}$ is discontinuous at $t_{k}$, although $\dot{V}_{2} \leq-\gamma\left[\left\|\left(\tilde{x}_{2}, \dot{\widetilde{x}}_{2}\right)\right\|\right]$ on $\left(t_{k}, t_{k+1}\right)$ for all $k \geq 0$. Indeed one obtains

$$
\sigma_{V_{2}}\left(t_{k}\right)=\sigma_{\dot{x}_{2}}\left(t_{k}\right)^{T}\left[\frac{\dot{x}_{2}\left(t_{k}^{+}\right)+\dot{x}_{2}\left(t_{k}^{-}\right)}{2}-c \tilde{x}_{2}\left(t_{k}\right)-\dot{x}_{2 d}\left(t_{k}\right)\right]
$$

which has no reason to be negative [see (63)]. Note that $\sigma_{V_{2}}\left(t_{k}\right)=f\left[\dot{x}_{2}\left(t_{k}^{+}\right), \tilde{x}_{2}\left(t_{k}\right)\right] \Delta_{k} \triangleq f_{k} \Delta_{k}$. Recall that from weak stability conditions, one must guarantee that $V\left(t_{0}^{k}\right) \geq$ $V\left(t_{f}^{k}\right)$. Due to the jumps in $V_{2}$ during the rebounds phase, it is not obvious whether there exists a time $t_{f}^{k}<+\infty$ such that this condition is satisfied. Indeed as we noted in Remark 7 , if the system remains long enough in $\Omega_{2 k}$, then $V\left(t_{0}^{k}\right)$ may be arbitrarily small. But $V_{2}\left(t_{\infty}\right)$ can be strictly positive because of some positive jumps. Notice that $V_{2}\left(t_{k+1}^{-}\right)-$ $V_{2}\left(t_{k}^{+}\right)=-\int_{\left(t_{k}, t_{k+1}\right)} \beta\left[\lambda_{1}, \lambda_{2}, \tilde{x}_{2}\left(t_{k}\right), \dot{\tilde{x}}_{2}\left(t_{k}^{+}\right), a, t\right] d t$ for some function $\beta \geq 0$. We therefore have at our disposal three parameters: $a, \lambda_{1}, \lambda_{2}$. The first one can be used to decrease the flight-times $\Delta_{k}$, whereas the other two control the variation of $V_{2}$ during flight-times. Assume that there exists $a^{\star}<+\infty$ $\lambda_{1}^{\star}<+\infty$ and $\lambda_{2}^{\star}<+\infty$ such that $\lambda_{1} \geq \lambda_{1}^{\star}, \lambda_{2} \geq \lambda_{2}^{\star}$, and $a>a^{\star}$ implies $\min _{t_{k}<t<t_{k+1}} \beta(t) \geq\left|f_{k}\right|$ for all $k \geq 0$. This insures that despite of possible positive jumps, $V_{2}$ decreases enough between the collisions so that $V_{2}\left(t_{k+1}^{-}\right) \leq V_{2}\left(t_{k}^{-}\right)$. Then existence of $t_{f}^{k}$ such that $V\left(t_{0}^{k}\right) \geq V\left(t_{f}^{k}\right)$ is guaranteed, and its value does not depend on the value of $V\left(t_{0}^{k}\right)$. This has important practical consequences since it means that the length of the different phases can be chosen independently, one from each other. Such conditions are evidently sufficient only to prove stability of the task. For the sake of briefness of the paper and since our goal is mainly to show which problems 
are posed by the stabilization of a complete task, we do not investigate the calculations more deeply.

In conclusion, unless $\mathcal{M}$ is a Jacobian and the closed-loop decoupled system (56) is used, there is no proper decoupling at the collision instants $t_{k}$ between "normal" and "tangential" components. Then the stability of a complete task is more difficult to obtain. But the conditions for weak stability in Lemma 1 are flexible enough to cope with such nondecoupled cases. This constitutes the main discrepancy between robotic tasks involving several regimes of motion and simple tasks and is a consequence of rigid body generalized impacts.

\section{CONCLUSIONS}

In this paper, we have studied the control of a class of mechanical systems subject to unilateral constraints on the position. We have reviewed the basic facts on the dynamics of such systems which are complex hybrid dynamical systems merging ordinary and measure differential equations, algebraic constraints, and possibly some high-level control strategy. In particular, attention has been focused on the definition and existence of the solutions of the closed-loop system as functions of local bounded variation in time and on the definition of the restitution rules. We have proposed a stability analysis framework that mixes and adapts some basic stability analysis for measure differential equations, hybrid dynamical systems and discrete-time Poincaré impact maps. This allowed us to analyze various discontinuous control strategies and is expected to provide a convenient stability analysis framework for subsequent extension of the results toward other controllers (such as adaptive controllers) and for robustness analysis (such as bad-timing effects for the switching times between different controllers, or bad knowledge of the constraint position). First we have focused on a one-dof example, and we have introduced various controllers to illustrate the different types of stability criteria we have proposed. Then we have discussed the $n$-dof case, and we have highlighted some difficulties related to the possible nondecoupling between the "normal" and the "tangential" parts of the velocities during the rebounds. We hope that the developed tools provide a first satisfactory theoretical answer to Professor Paul's statement [40] that "the contact problem is unsolved for rigid manipulator, rigid sensor, rigid environment problems," that is naturally embedded into the class of systems with unilateral constraints. Finally, this work is to the authors' knowledge the first extension of force/position control of rigid manipulators subject to holonomic constraints $F(q)=0$ (McClamroch-Wang [5] and Yoshikawa [7]) to the case of unilateral constraints $F(q) \geq 0$ that takes into account all the problems related to mechanics, mathematics, and closed-loop stability.

\section{APPENDIX PROOF OF LEMMA 1}

Case a: Assume that $\lim _{t \rightarrow \infty, t \in \Omega} V[x(t)]=\delta>0$. Since $\beta(\|x\|) \geq V(x)$ for all $t \geq 0$, and $\mathrm{V}$ is nonincreasing on $\Omega$, then $\bar{\delta}=\min _{t \in \Omega} V(t)$, and $\|x(t)\| \geq \beta^{-1}(\delta)>0$ for all $t \in \Omega$. Since $t \in \Omega$, we can write for a certain $n \in \mathbb{N}$ (assuming the initial time is in $\Omega$ ):

$$
\begin{aligned}
V(t)-V(0)= & V(t)-V\left(t_{f}^{n}\right)+V\left(t_{f}^{n}\right)-V\left(t_{0}^{n}\right)+V\left(t_{0}^{n}\right) \\
& -V\left(t_{f}^{n-1}\right)+V\left(t_{f}^{n-1}\right)-V\left(t_{0}^{n-1}\right) \\
& +V\left(t_{0}^{n-1}\right)+\cdots+V\left(t_{f}^{0}\right)-V(0)
\end{aligned}
$$

Consequently, since $V\left(t_{f}^{k}\right) \leq V\left(t_{0}^{k}\right)$ we obtain

$$
\begin{aligned}
V(t)-V(0) \leq & -\sum_{j=1}^{n} \int_{t_{f}^{j-1}}^{t_{f}^{j}} \gamma[\|x(\tau)\|] d \tau \\
& -\int_{t_{f}^{n}}^{t} \gamma[\|x(\tau)\|] d \tau \\
& -\int_{0}^{t_{f}^{0}} \gamma[\|x(\tau)\|] d \tau+\sum_{k} \sigma_{V}\left(t_{k}\right) \\
\leq- & \gamma_{0} \beta^{-1}(\delta) \lambda[\Omega[0, t]] .
\end{aligned}
$$

Now we can always find $t$ (or $n$ ) such that for any $V(0)<+\infty$ and for any $\delta>0$ the inequality $V(t)+\gamma_{0} \beta^{-1}(\delta) \lambda\left[\Omega_{[0, t]}\right] \leq$ $V(0)$ is not satisfied. Hence by contradiction we deduce that $\delta=0$, and since $\|x(t)\| \leq \alpha^{-1} \circ V[x(t)]$ it follows that $\lim _{\{t \rightarrow \infty, t \in \Omega\}} x(t)=0$. Note that if $t \notin \Omega$, the reasoning fails because for $t \in I$ the terms $V(t)-V\left(t_{0}^{k}\right)$ may be positive.

Case $b$ : The case b) can be similarly proved by noting that $\|x(t)\| \geq \beta^{-1}(\delta)>0$ for all $t \in \Omega$ implies that necessarily $\left\|\xi_{1}\right\| \geq \delta_{1}>0$ and/or $\left\|\xi_{2}\right\| \geq \delta_{2}>0$ for some $\delta_{1}$ and $\delta_{2}$. Then, using that $\left\|\xi_{1}\right\|$ and $\left\|\xi_{2}\right\|$ do not increase on $\Omega^{2}$ and $\Omega^{1}$, respectively, and that these time intervals have infinite Lebesgue measure, one deduces that if either $\delta_{1}$ or $\delta_{2}$ is strictly positive (i.e., $\delta>0$ ), then a contradiction appears and necessarily $\delta=0$.

Case c: From the assumptions there exists a time $T<+\infty$ such that for all $t \geq T, \xi_{1}(t)$ is a constant vector $X_{1}$. Let us define the function $V_{X_{1}}\left(\xi_{2}\right) \triangleq V\left(X_{1}, \xi_{2}\right)-V\left(X_{1}, 0\right)$. Then for any $\delta \geq 0$ the sets $V_{X_{1}}^{-1}(\delta)=\left\{\xi_{2}: V_{X_{1}}\left(\xi_{2}\right) \leq \delta\right\}$ are equal to the sets $V^{-1}\left[\delta+V\left(X_{1}, 0\right)\right] \cap\left\{\xi_{1}=X_{1}\right\} \subset \mathbb{R}^{n}$. Hence they are bounded and closed, thus compact. Now $V_{X_{1}}(0)=0, V_{X_{1}}\left(\xi_{2}\right) \geq 0, \dot{V}_{X_{1}}(t)=\dot{V}(t) \leq-\gamma_{2}\left(\left\|\xi_{2}(t)\right\|\right)$ for $t \neq t_{k}$, and $\sigma_{V_{X_{1}}}\left(t_{k}\right)=\sigma_{V} \leq 0$. The conclusion follows similarly as in a). The conditions apply when for instance $V\left(\xi_{1}, \xi_{2}\right)=V_{1}\left(\xi_{1}\right)+V_{2}\left(\xi_{2}\right), V_{1}\left(\xi_{1}\right) \geq 0, V_{2}\left(\xi_{2}\right) \geq 0$.

\section{REFERENCES}

[1] D. D. Bainov and P. S. Simeonov, Systems with Impulse Effect: Stability, Theory and Applications. New York: Wiley, 1989.

[2] M. S. Branicky, "Stability of switched and hybrid systems," in Proc. IEEE Conf. Dec. Contr., Lake Buena Vista, FL, 1994.

[3] M. S. Branicky, V. S. Borkar, and S. K. Mitter, "A unified framework for hybrid control: Background, model and theory," in 11th Int. Conf. Analysis Optimization Syst., INRIA, France, June 15-17, 1994, LIDSP-2239, MIT, revised.

[4] B. Brogliato, P. Orhant, and R. Lozano, "On the transition phase in robotics-Part 1: Impacts models and dynamics; Part 2: Control" in Proc. IFAC Syroco, Capri, Italy, pp. 565-576, Sept. 1994.

[5] N. H. McClamroch and D. Wang, "Feedback stabilization and tracking of constrained robots," IEEE Trans. Automat. Contr., vol. 33, pp. 419-426, 1988

[6] G. T. Marth, T. J. Tarn, and A. K. Bejczy, "Stable transition phase for robot arm control," in Proc. IEEE Int. Conf. Robotics Automation, Atlanta, GA, 1993, pp. 355-362. 
[7] T. Yoshikawa, "Dynamic hybrid position/force control of robot manipulators-Description of hand constraints and calculation of joint driving force," IEEE J. Robotics Automation, vol. 3, pp. 386-392, 1987.

[8] R. M. Brach, Mechanical Impact Dynamics. New York: Wiley, 1991

[9] L. Paoli and M. Schatzman, "Mouvement à un nombre fini de degrés de liberté avec contraintes unilatérales," Math. Model. Num. Anal. (M2AN), vol. 27, no. 6, pp. 673-717, 1993

[10] L. Paoli, "Analyze numérique de vibrations avec contraintes unilatérales," Ph.D. dissertation, Univ. C. Bernard-Lyon 1, Laboratoire d'Analyze numérique, URA CNRS 740, 1993.

[11] L. Paoli and M. Schatzman, private correspondence, 1995.

[12] A. P. Ivanov, "Impacts in a system with certain unilateral couplings," Prikl. Matem. Mekhan., vol. 51, no. 4, pp. 559-566, 1987; translated in J. Appl. Math. Mechanics, vol. 51, no. 4, pp. 436-442, 1988.

[13] V. V. Kozlov and D. V. Treshchev, Billiards-A Genetic Introduction to the Dynamics of Systems with Impacts. Providence, RI: Amer. Math. Soc., 1991.

[14] J. J. Moreau, "Unilateral contact and dry friction in finite freedom dynamics," in Nonsmooth Mechanics Appl., CISM Courses Lectures, no. 302. New York: Springer-Verlag, 1988, pp. 1-82.

[15] M. D. P. Monteiro-Marques, Differential Inclusions in Nonsmooth $\mathrm{Me}$ chanical Problems: Shocks and Dry Friction. Boston: Birkhauser, 1993, PNLDE 9.

[16] J. K. Mills and D. M. Lokhorst, "Control of robotic manipulators during general task execution: A discontinuous control approach," Int J. Robotics Res., vol. 12, no. 2, pp. 146-163, 1993.

[17] J. K. Mills, "Stability of robotic manipulators during transition to and from compliant motion," Automatica, vol. 26, no. 5, pp. 861-874, 1990

[18] J. K. Mills and D. M. Lokhorst, "Stability and control of robotic manipulators during contact/noncontact task transition," IEEE Trans. Robotics Automation, vol. 9, pp. 335-345, June 1993.

[19] G. T. Marth, T. J. Tarn, and A. K. Bejczy, "An event based approach to impact control: Theory and experiments," in IEEE Int. Conf. Robotics Automation, San Diego, CA, 1994, pp. 918-923.

[20] O. Khatib and J. Burdick, "Motion and force control of robot manipulators," in IEEE Int. Conf. Robotics Automation, San Francisco, CA, 1986, pp. 1381-1386.

[21] K. Youcef-Toumi and D. A. Gutz, "Impact and force control: Modeling and experiments," ASME J. Dyn. Syst. Meas. Contr., vol. 116, pp. 89-98, Mar. 1994.

[22] J. M. Hyde and M. R. Cutkosky, "Contact transition control: An experimental study," IEEE Contr. Syst., vol. 14, no. 1, pp. 25-31, Feb. 1994.

[23] D. M. Lokhorst and J. K. Mills, "Implementation of a discontinuous control law on a robot during collision with a stiff environment," in Proc. IEEE Conf. Robotics Automation, Cincinnati, OH, 1990.

[24] L. Cai and A. A. Goldenberg, "Robust control of position and force for robot manipulators involving both free and constrained motion," in Proc. IEEE Conf. Decision Contr., Honolulu, HI, 1990, pp. 1943-1948.

[25] M. Schatzman, "A class of nonlinear differential equations of second order in time," Nonlinear Analysis, Theory, Methods, Applications, vol. 2, no. 3, pp. 355-373, 1978.

[26] D. Percivale, "Uniqueness in the elastic bounce problem," J. Differential Equations, vol. 56, pp. 206-215, 1985; also vol. 90, pp. 304-315, 1991.

[27] R. Abraham and J. E. Mardsen, Foundations of Mechanics. New York: Addison-Wesley, 1978.

[28] M. P. Wojtkowski, "A system of one dimensional balls with gravity," Comm. Math. Phys., vol. 126, pp. 507-533, 1990.

[29] J. J. Slotine and W. Li, "Adaptive manipulator control: A case study," IEEE Trans. Automat. Contr., vol. 33, pp. 995-1003, 1988.

[30] M. W. Spong and M. Vidyasagar, Robot Dynamics and Control. New York: Wiley, 1989.

[31] B. Brogliato and P. Orhant, "On the control of the transition phase in robotics: Impact models, dynamics and control," in IEEE Int. Conf. Robotics Automation, San Diego, CA 1994.

[32] X. Liu, "Stability theory for impulsive differential equations in terms of two measures," Lectures Notes in Pure and Applied Mathematics. New York: Marcel Dekker, 1991, vol. 127.

[33] V. I. Zubov, Methods of A. M. Lyapunov and Their Applications. The Netherlands: Nordhoff, 1964

[34] A. F. Filipov, Differential Equations with Discontinuous Right-HandSides. Dordercht, The Netherlands: Kluwer, 1988.

[35] Y. Wang, "Dynamic modeling and stability analysis of mechanical systems with time-varying topologies," ASME J. Mechanical Design vol. 115 , pp. 808-816, Dec. 1993

[36] A. I. Volpert and S. I. Hudjaev, Analysis in Classes of Discontinuous Functions and Equations of Mathematical Physics. Dordrecht: Nijhoff, 1985.
[37] L. B. Rapoport, "Stability of the equilibrium of a system with singlesided constraints and the sign-definiteness of a pencil of quadratic forms in a cone," Prikl. Matem. Mekhan., vol. 56, no. 4, pp. 598-604, 1992; Translated in J. Appl. Math. Mechanics, vol. 56, no. 4, pp. 501-507, 1992.

[38] A. P. Ivanov,"Analytical methods in the theory of vibro impact systems,"Prikl. Matem. Mekhan., vol.57,no.2,pp. 5-21,1993; Translated in J. Appl. Math. Mechanics, vol.57,no 2,pp.221-236,1993.

[39] K. Gajewski and B. Radziszewski, "On the stability of impact systems," Bull. Polish Acad. Sci., Technical Sci., Appl. Mechanics, vol. 35, nos. 3 and 4, pp. 183-189, 1987.

[40] R. P. Paul, "Problems and research issues associated with the hybrid control of force and displacements," IEEE Int. Conf. Robotics Automation, 1987, pp. 1966-1971.

[41] Y. Hurmuzlu and G. D. Moskowitz, "Bipedal locomotion stabilized by impact and switching:- I: Two and three-dimensional, three element models; II: Structural stability analysis of a four element bipedal locomotion," Dynamics Stability Syst., vol. 2, no. 2, pp. 73-112, 1987.

[42] F. Pierrot, M. Jean, and P. Dauchez, "Nonsmooth mechanics approach for robot simulations," in Proc. IFAC SYROCO, Capri, Italy, Sept. 1994, preprints, pp. $577-582$

[43] D. Stoianovici and Y. Hurmuzlu, "A critical study of the applicability of rigid body collision theory," ASME J. Appl. Mechanics, 1996.

[44] R. Volpe and P. Khosla, "A theoretical and experimental investigation of impact control for manipulators," Int. J. Robotics Res., vol. 12, no. 4, pp. 351-365, 1993

[45] P. Pagilla and M. Tomizuka, "Control of mechanical systems subject to unilateral constraints," IEEE Conf. Decision Contr., New Orleans, LA, Dec. 13-15, 1995

[46] Y. Hurmuzlu and T. H. Chang, "Rigid body collisions of a special class of planar kinematic chains," IEEE Trans. Syst., Man Cybern., vol. 22, pp. 964-971, Sept./Oct. 1992.

[47] Y. Hurmuzlu and D. B. Marghitu, "Rigid body collision of planar kinematic chains with multiple contact points," Int. J. Robotics Res., vol. 13, pp. 82-92, Feb. 1994

[48] Y. F. Zheng and H. Hemami, "Mathematical modeling of a robot collision with its environment," J. Robotic Syst., vol. 2, no. 3, pp. 289-307, 1985

[49] B. Chapnik, G. Heppler, and J. Aplevich, "Modeling impact on a onelink flexible robotic arm," IEEE Trans. Robotics Automation, vol. 7, pp. 479-488, Aug. 1991

[50] A. Tornambé, "Global regulation of planar robot arm striking a surface," IEEE Trans. Autom. Contr., Sept. 1995.

[51] _ "Modeling and controlling one-degree-of-freedom impacts," IEE Proc. Control Theory Appl., vol. 143, no. 1, pp. 85-90, 1996.

[52] J. R. Kalagnanam, "Controlling chaos: The example of an impact oscillator," ASME J. Dyn. Syst., Meas. Contr., vol. 116, pp. 557-564, Sept. 1994

[53] T. L. Vincent, "Controlling the bouncing ball to fixed points corresponding to bounce cycles," in Am. Contr. Conf., Seattle, WA, June 1995, pp. 842-846.

[54] M. Buhler, D. E. Koditschek, and P. J. Kindlmann, "A family of robo control strategies for intermittent dynamical environments," IEEE Contr. Syst. Mag., pp. 16-22, Feb. 1990.

[55] M. W. Gertz, J. O. Kim, and P. K. Khosla, "Exploiting redundancy to reduce impact force," in IEEE/RSJ Int. Wkshp. Intelligent Robots Syst., IROS 91, Osaka, Japan, Nov. 1991, pp. 179-184.

[56] K. Yoshida, "Impact dynamics representation and control with extendedinversed inertia tensor for space manipulators," Robotics Res. 6, T. Kanade and R. Paul, Eds. Cambridge: IFRR, 1994, pp. 453-463.

[57] N. Mandal and S. Payandeh, "Control strategies for robotic tasks: An experimental study," J. Robotic Syst., vol. 12, no. 1, pp. 67-92, 1995.

[58] H. Kazerooni, B. J. Waibel, and S. Kim, "On the stability of robot compliant motion control: Theory and experiments," ASME J. Dyn. Syst. Meas. Contr., vol. 112, pp. 417-426, 1990.

[59] N. Mandal and S. Payandeh, "Experimental evaluation of the importance of compliance for robotic impact control," in IEEE Contr. Appl. Conf., 1993, pp. 511-516.

[60] P. Lotstedt, "Mechanical systems of rigid bodies subject to unilateral constraints," SIAM J. Appl. Math., vol. 42, pp. 281-296, 1982.

[61] _ "Numerical simulation of time dependent contact and friction problems in rigid body mechanics," SIAM J. Sci. Stat. Comput., vol. 5, pp. 370-393, 1984

[62] H. P. Huang and N. H. McClamroch, "Time optimal control of a robotic contour following problem," IEEE J. Robotics Automation, vol. 4, pp. 140-149, 1988

[63] A. J. van der Schaft and J. M. Schumacher, “ The complementarity -slackness class of hybrid systems," Memo. no. 1290, 
ISSN 0169-2690, University of Twente, Nov. 1995; Math. Contr., Signal Syst., submitted.

[64] B. Brogliato, Nonsmooth Impact Mechanics. Models, Dynamics and Control. New York: Springer Verlag, 1996, LNCIS 220. 